\title{
Formation of Tetrahydrothiophenes via a Thia-Paternò-Büchi Initiated Domino Photochemical Reaction
}

\author{
Ahmad F. Kassir, Régis Guillot, Marie-Christine Scherrmann, Thomas Boddaert, ${ }^{*}$ and \\ David J. Aitken*
}

Université Paris-Saclay, CNRS, Institut de Chimie Moléculaire et des Matériaux d'Orsay (ICMMO), 91405, Orsay, France.

E-mail : thomas.boddaert@universite-paris-saclay.fr, david.aitken@universite-paris-saclay.fr

\section{Supporting Information}

1. General experimental:

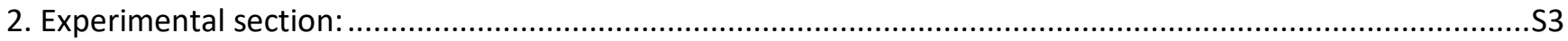

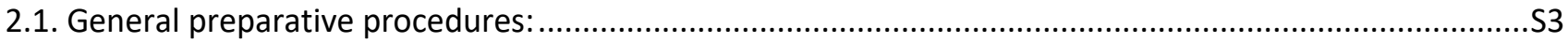

2.2. Preparation of "homo" tetra-aryl tetrahydrothiophenes 4a-e from thioketones 1a-e:............................S4

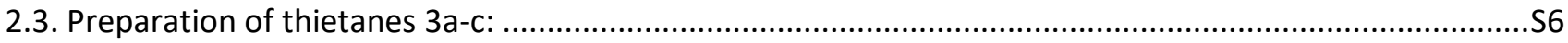

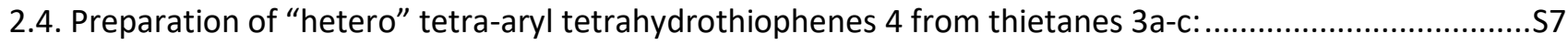

2.5. Procedures and analytical data for additional experiments: .................................................................

3. ${ }^{1} \mathrm{H}$ and ${ }^{13} \mathrm{CNMR}$ spectra of "homo" tetra-aryl tetrahydrothiophenes 4a-e (Scheme 1): ..............................S16

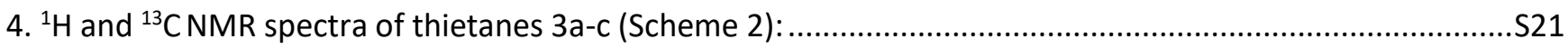

5. ${ }^{1} \mathrm{H}$ and ${ }^{13} \mathrm{C}$ NMR spectra of "hetero" tetra-aryl tetrahydrothiophenes 4 (Scheme 3): .................................S24

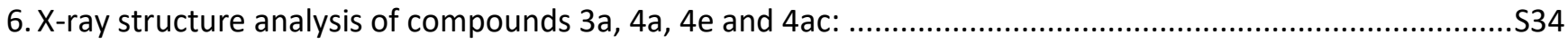




\section{General experimental:}

Solvents were of commercial grade and were used without further purification with the exception of dichloromethane which was dried over activated alumina, tetrahydrofuran which was distilled from sodium/benzophenone, cyclohexane which was distilled from $\mathrm{P}_{2} \mathrm{O}_{5}$, toluene and benzene which were both distilled from $\mathrm{CaH}_{2}$. All reagents were used as received, with the exception of acrylonitrile which was fresly distilled under argon atmosphere immediately before use.

Flash chromatography was performed on 35-70 $\mu \mathrm{m}$ columns of silica gel (Merck-Chimie SAS). Analytical thinlayer chromatography was carried out on commercial $0.25 \mathrm{~mm}$ silica gel plates (EMD, Silica Gel 60F254) which were visualized by UV fluorescence at $254 \mathrm{~nm}$ and then revealed using a phosphomolybdic acid solution (10\% in $\mathrm{EtOH})$ or a ninhydrin solution $(0.3 \%$ in $n-\mathrm{BuOH})$. Retention factors $\left(\mathrm{R}_{\mathrm{f}}\right)$ are given for such TLC analyses. Melting points were determined with a Büchi B-545 apparatus in open capillary tubes and are uncorrected. Fouriertransform infrared (IR) spectra were recorded for neat liquid or solid samples on a PerkinElmer Spectrum Two spectrophotometer using an ATR diamond accessory; maximum absorbances $(\mathrm{v})$ are given in $\mathrm{cm}^{-1}$. Nuclear magnetic resonance (NMR) data were acquired on Bruker spectrometers operating at 400/360/300/250 MHz for ${ }^{1} \mathrm{H}$, at $100 / 90 / 75 / 63 \mathrm{MHz}$ for ${ }^{13} \mathrm{C}$ and at $235 \mathrm{MHz}$ for ${ }^{19} \mathrm{~F}$. Chemical shifts $(\delta)$ are reported in ppm with respect to the residual proton signal in deuterated chloroform $(\delta=7.26 \mathrm{ppm})$ for ${ }^{1} \mathrm{H}$, with respect to $\mathrm{CDCl}_{3}(\delta=77.16 \mathrm{ppm})$ for ${ }^{13} \mathrm{C}$ and with respect to $\mathrm{CFCl}_{3}(\delta=0 \mathrm{ppm})$ for ${ }^{19} \mathrm{~F}$. Splitting patterns for ${ }^{1} \mathrm{H}$ and ${ }^{13} \mathrm{C}$ NMR signals are designated as s (singlet), d (doublet), t (triplet), app. (apparent), or $\mathrm{m}$ (multiplet). Coupling constants $(\mathrm{J})$ are reported in $\mathrm{Hz}$. High-resolution mass spectrometry (HRMS) data were recorded on a Bruker Daltonics MicroTOF-Q spectrometer equipped with an electrospray ionization source in positive mode, with a tandem Q-TOF analyzer. Details of the X-ray diffraction analyses are described in section 6.

Photochemical reactions using polychromatic light were performed using a $400 \mathrm{~W}$ medium-pressure $\mathrm{Hg}$ lamp fitted with a Pyrex filter and a water-cooling jacket. Photochemical reactions using monochromatic light (300 or $350 \mathrm{~nm}$ lamps) were performed using a Rayonet RPR-200 photochemical reactor equipped with 16 fluorescent lamps: either RPR-3000 $\AA$ ( $21 \mathrm{~W}$; emission maximum $\lambda=300 \mathrm{~nm}$; half-intensity emission range 285-320 nm) or RPR-3500 $\AA$ ( $24 \mathrm{~W}$; emission maximum $\lambda=350 \mathrm{~nm}$; half-intensity emission range 330-370 nm). 


\section{Experimental section:}

\subsection{General preparative procedures:}

General experimental procedure A for the domino photochemical reaction:

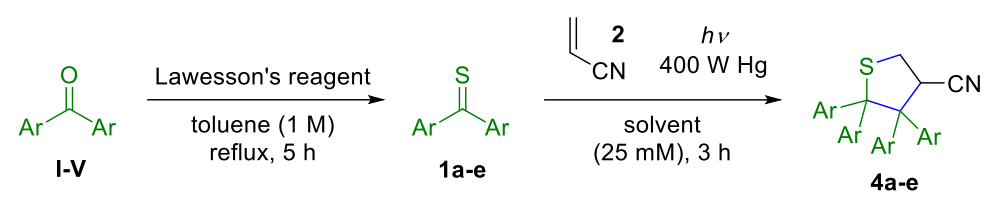

Ketone I-V (1 equiv.) was dissolved in toluene $(1 \mathrm{~mL} / \mathrm{mmol}$ ) and degassed with an argon stream for 10 minutes. Lawesson's reagent ( 0.7 equiv.) was added to the reaction mixture and refluxed for $5 \mathrm{~h}$ under argon using an oil bath. The reaction mixture was then cooled to room temperature and toluene was evaporated under reduced pressure. The crude thioketone 1a-e was dissolved directly in cyclohexane $(40 \mathrm{~mL} / \mathrm{mmol})$ then acrylonitrile 2 (23 equiv.) was added. The reaction mixture was placed in a cylindrical immersion photochemical reactor and degassed with an argon stream for 15 minutes. The solution was irradiated for $3 \mathrm{~h}$ with a $400 \mathrm{~W}$ medium-pressure Hg lamp fitted with a Pyrex filter and a water-cooling jacket. The reaction mixture was then evaporated under reduced pressure to give the crude product, which was purified by flash chromatography to afford the requisite tetrahydrothiophene product 4a-e. (See Table 1 and Scheme 1, manuscript)

\section{General experimental procedure B for the thia-Paternò-Büchi reaction:}

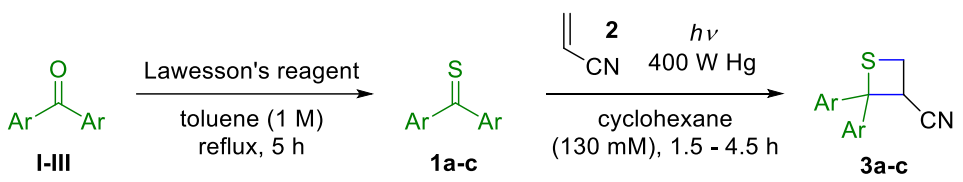

Ketone I-III (1 equiv.) was dissolved in toluene $(1 \mathrm{~mL} / \mathrm{mmol})$ and degassed with an argon stream for 10 minutes. Lawesson's reagent ( 0.7 equiv.) was added to the reaction mixture and refluxed for $5 \mathrm{~h}$ under argon using an oil bath. The reaction mixture was then cooled to room temperature and toluene was evaporated under reduced pressure. The crude thioketone 1a-c was dissolved directly in cyclohexane $(6.2 \mathrm{~mL} / \mathrm{mmol})$ then acrylonitrile $2(23$ equiv.) was added. The reaction mixture was placed in a Pyrex tube and degassed with an argon stream for 15 minutes. The solution was irradiated for the specified time with a $400 \mathrm{~W}$ medium-pressure $\mathrm{Hg}$ lamp fitted with a Pyrex filter and a water-cooling jacket. The reaction mixture was then evaporated under reduced pressure to give the crude product, which was purified by flash chromatography to afford the requisite thietane product 3ac (see Table 1 and Scheme 2, manuscript). 
General experimental procedure $\mathrm{C}$ for the photochemical ring enlargement:

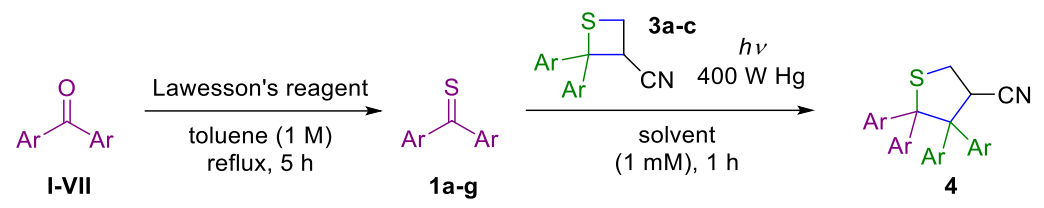

Ketone I-VII (2 or 3 equiv.) was dissolved in toluene $(1 \mathrm{~mL} / \mathrm{mmol}$ ) and degassed with an argon stream for 10 minutes. Lawesson's reagent (1.4 or 2.1 equiv.) was added to the reaction mixture and refluxed for $5 \mathrm{~h}$ under argon using an oil bath. The reaction mixture was then cooled to room temperature and toluene was evaporated under reduced pressure. The crude thioketone 1a-g was dissolved directly in cyclohexane $(1 \mathrm{~L} / \mathrm{mmol})$ then thietane 3a-c (1 equiv.) dissovled in few drops of dichloromethane was added. The reaction mixture was placed in a cylindrical immersion photochemical reactor and degassed with an argon stream for 15 minutes. The solution was irradiated for $1 \mathrm{~h}$ with a $400 \mathrm{~W}$ medium-pressure Hg lamp fitted with a Pyrex filter and a water-cooling jacket. The reaction mixture was then evaporated under reduced pressure to give the crude product, which was purified by flash chromatography to afford the requisite tetrahydrothiophene product 4 (see Table 2 and Scheme 3, manuscript).

\subsection{Preparation of "homo" tetra-aryl tetrahydrothiophenes 4a-e from thioketones 1a-e:}

\section{4,4,5,5-Tetraphenyltetrahydrothiophene-3-carbonitrile 4a}

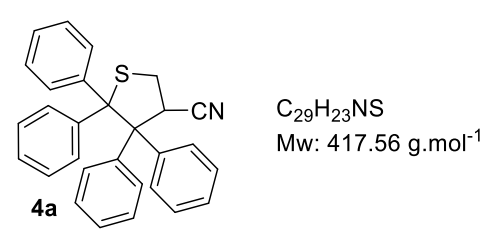

General procedure A was applied to benzophenone I (911 mg, $5.0 \mathrm{mmol}$ ) and acrylonitrile $2(7.57 \mathrm{~mL}, 115.0 \mathrm{mmol})$ in cyclohexane $(200 \mathrm{~mL})$. Flash chromatography (01:99 EtOAc/Petroleum ether to 10:90 EtOAc/Petroleum ether) gave product $4 \mathrm{a}$ as a white powder (650 $\mathrm{mg}, 62 \%)$.

$\mathbf{R}_{\mathbf{f}}\left(\mathbf{1 0 : 9 0}\right.$ EtOAc/Petroleum ether): 0.52. Mp: $224-225^{\circ} \mathrm{C}$. IR v (cm $\left.{ }^{-1}\right): 3052,2243,1596,1487,1439,1199,1035$, 743, 725, 701. ${ }^{1} \mathrm{H}$ NMR (360 MHz, $\left.\mathrm{CDCl}_{3}\right): \delta 7.97(\mathrm{~d}, J=7.6 \mathrm{~Hz}, 2 \mathrm{H}), 7.42-6.87(\mathrm{~m}, 18 \mathrm{H}), 4.47(\mathrm{dd}, J=12.0,6.6 \mathrm{~Hz}$, 1H), 3.42 (dd, $J=10.3,6.6 \mathrm{~Hz}, 1 \mathrm{H}$ ), 2.92 (dd, $J=12.0,10.3 \mathrm{~Hz}, 1 \mathrm{H}) .{ }^{13} \mathrm{C} \mathrm{NMR}\left(90 \mathrm{MHz}, \mathrm{CDCl}_{3}\right): \delta 146.1$ (C), 143.8 (C), $139.2(\mathrm{C}), 137.8(\mathrm{C}), 132.5(2 \mathrm{CH}), 131.0(2 \mathrm{CH}), 130.4(2 \mathrm{CH}), 129.9(2 \mathrm{CH}), 128.0(\mathrm{CH}), 127.7(2 \mathrm{CH}), 127.4(\mathrm{CH})$, 127.2 (2CH), $127.1(2 \mathrm{CH}), 126.52(2 \mathrm{CH}), 126.48(2 \mathrm{CH}), 118.2(\mathrm{C}), 73.1(\mathrm{C}), 66.7(\mathrm{C}), 41.8(\mathrm{CH}), 30.4\left(\mathrm{CH}_{2}\right)$. HRMS (ESI-TOF) $m / z:[\mathrm{M}+\mathrm{Na}]^{+}$Calcd for $\mathrm{C}_{29} \mathrm{H}_{23} \mathrm{NSNa} 440.1449$. Found 440.1426 .

\section{4,4,5,5-Tetra-p-tolyltetrahydrothiophene-3-carbonitrile 4b}

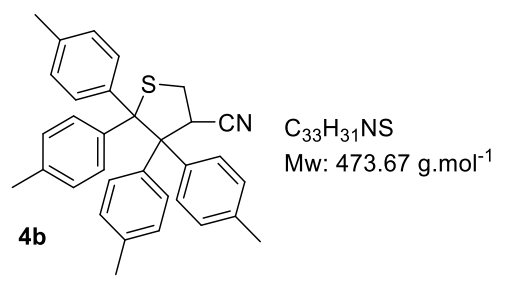

General procedure A was applied to 4,4'-dimethylbenzophenone II (959 mg, $4.56 \mathrm{mmol}$ ) and acrylonitrile $2(6.9 \mathrm{~mL}, 104.9 \mathrm{mmol})$ in cyclohexane $(200 \mathrm{~mL})$. Flash chromatography (01:99 EtOAc/Petroleum ether to 05:95 EtOAc/Petroleum ether) gave product $\mathbf{4 b}$ as a white powder (598 mg, 55\%). 
$\mathbf{R}_{\mathrm{f}}$ (04:96 EtOAc/Petroleum Ether): 0.21. Mp: 216-217 ${ }^{\circ} \mathrm{C}$. IR v (cm $\left.{ }^{-1}\right): 3025,2919,2863,2236,1507,1444,1191$, 1020, 803, 762. ${ }^{1} \mathrm{H}$ NMR (400 MHz, $\left.\mathrm{CDCl}_{3}\right): \delta 7.81(\mathrm{~d}, J=8.1 \mathrm{~Hz}, 2 \mathrm{H}), 7.18(\mathrm{~d}, J=8.1 \mathrm{~Hz}, 2 \mathrm{H}), 7.08(\mathrm{~d}, J=8.3 \mathrm{~Hz}$, $2 \mathrm{H}), 6.95-6.70(\mathrm{~m}, 10 \mathrm{H}), 4.36(\mathrm{dd}, J=12.0,6.5 \mathrm{~Hz}, 1 \mathrm{H}), 3.34(\mathrm{dd}, J=9.9,6.5 \mathrm{~Hz}, 1 \mathrm{H}), 2.88-2.80(\mathrm{~m}, 1 \mathrm{H}), 2.36(\mathrm{~s}$, 3H), $2.26(\mathrm{~s}, 3 \mathrm{H}), 2.26(\mathrm{~s}, 3 \mathrm{H}), 2.23(\mathrm{~s}, 3 \mathrm{H}) .{ }^{13} \mathrm{C} \mathrm{NMR}\left(63 \mathrm{MHz}, \mathrm{CDCl}_{3}\right)$ : $\delta 143.6$ (C), 141.1 (C), 137.5 (C), 137.1 (C), $136.4(\mathrm{C}), 136.1$ (C), 136.0 (C), $135.2(\mathrm{C}), 132.5(2 \mathrm{CH}), 131.0(2 \mathrm{CH}), 130.2(2 \mathrm{CH}), 130.1(2 \mathrm{CH}), 128.2(2 \mathrm{CH}), 127.9$ $(2 \mathrm{CH}), 127.7(2 \mathrm{CH}), 127.1(2 \mathrm{CH}), 118.6(\mathrm{C}), 72.6(\mathrm{C}), 66.2(\mathrm{C}), 42.0(\mathrm{CH}), 30.3\left(\mathrm{CH}_{2}\right), 21.1\left(\mathrm{CH}_{3}\right), 21.0\left(\mathrm{CH}_{3}\right), 20.9$ $\left(\mathrm{CH}_{3}\right), 20.9\left(\mathrm{CH}_{3}\right)$. HRMS (ESI-TOF) $\mathrm{m} / \mathrm{z}$ : $[\mathrm{M}+\mathrm{Na}]^{+}$Calcd for $\mathrm{C}_{33} \mathrm{H}_{31} \mathrm{NSNa}$ 496.2069. Found 496.2096.

\section{4,4,5,5-Tetrakis(4-chlorophenyl)tetrahydrothiophene-3-carbonitrile 4c}

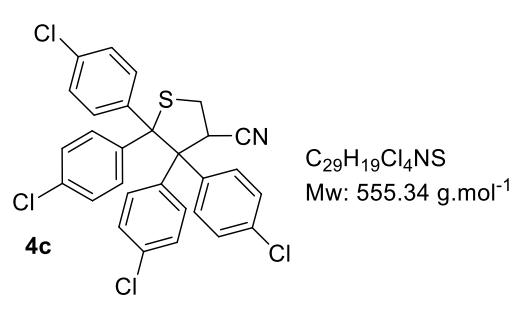

General procedure A was applied to 4,4'-dichlorobenzophenone III (1.143 g, $4.55 \mathrm{mmol}$ ) and acrylonitrile $2(6.89 \mathrm{~mL}, 104.65 \mathrm{mmol})$ in cyclohexane (200 $\mathrm{mL})$. Flash chromatography $\left(30: 70 \quad \mathrm{CH}_{2} \mathrm{Cl}_{2}\right.$ /Petroleum ether to 50:50 $\mathrm{CH}_{2} \mathrm{Cl}_{2} /$ Petroleum ether) gave product $4 \mathrm{c}$ as a white powder (651 $\left.\mathrm{mg}, 52 \%\right)$.

$\mathbf{R}_{\mathbf{f}}\left(40: 60 \mathrm{CH}_{2} \mathrm{Cl}_{2} /\right.$ Petroleum Ether): 0.51. Mp: $231-232{ }^{\circ} \mathrm{C}$. IR v $\left(\mathrm{cm}^{-1}\right): 3081$, 3062, 3025, 2939, 2242, 1591, 1490, 1398, 1095, 1011, 799, 780, 753. ${ }^{1} \mathbf{H}$ NMR (360 MHz, CDCl $): \delta 7.81$ (d, J = $8.6 \mathrm{~Hz}, 2 \mathrm{H}), 7.28(\mathrm{~d}, J=9.1 \mathrm{~Hz}, 2 \mathrm{H}), 7.22-7.09(\mathrm{~m}, 6 \mathrm{H}), 7.04-6.78(\mathrm{~m}, 6 \mathrm{H}), 4.25(\mathrm{dd}, J=11.9,6.6 \mathrm{~Hz}, 1 \mathrm{H}), 3.41$ (dd, $J=10.5,6.6 \mathrm{~Hz}, 1 \mathrm{H}$ ), 2.84 (dd, $J=11.9,10.5 \mathrm{~Hz}, 1 \mathrm{H}) .{ }^{13} \mathrm{C}$ NMR (63 MHz, CDCl 3 ): $\delta 143.9$ (C), 141.4 (C), 137.0 (C), 135.3 (C), 134.7 (C), 134.4 (C), $133.7(2 \mathrm{CH}), 133.6$ (C), 133.1 (C), $132.3(2 \mathrm{CH}), 131.3(2 \mathrm{CH}), 131.2(2 \mathrm{CH}), 128.3$ (2CH), $127.9(2 \mathrm{CH}), 127.7(2 \mathrm{CH}), 127.1(2 \mathrm{CH}), 117.3$ (C), 71.9 (C), 66.0 (C), $42.0(\mathrm{CH}), 30.3\left(\mathrm{CH}_{2}\right)$. HRMS (ESI-TOF) $\mathrm{m} / \mathrm{z}:[\mathrm{M}+\mathrm{Na}]^{+}$Calcd for $\mathrm{C}_{29} \mathrm{H}_{19}{ }^{35} \mathrm{Cl}_{4} \mathrm{NSNa}$ 575.9885. Found 575.9862.

\section{4,4,5,5-Tetrakis(4-methoxyphenyl)tetrahydrothiophene-3-carbonitrile 4d}

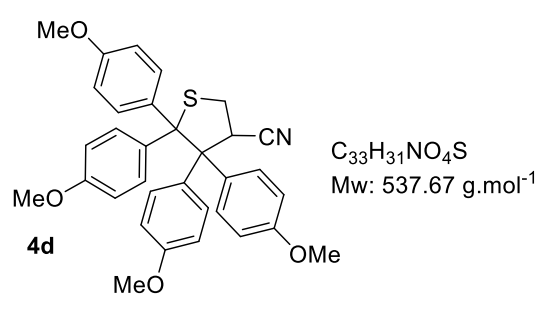

General procedure A was applied to 4,4'-dimethoxy benzophenone IV (1.187 g, $4.9 \mathrm{mmol})$ and acrylonitrile $2(7.42 \mathrm{~mL}, 112.7 \mathrm{mmol})$ in cyclohexane (200 mL). Flash chromatography (80:20 $\mathrm{CH}_{2} \mathrm{Cl}_{2}$ /Petroleum ether to 90:10 $\mathrm{CH}_{2} \mathrm{Cl}_{2} / \mathrm{EtOAc}$ ) gave product $4 \mathrm{~d}$ as a pale yellow powder (685 $\mathrm{mg}, 52 \%$ ).

$\mathbf{R}_{\mathrm{f}}$ (70:30 $\mathrm{CH}_{2} \mathrm{Cl}_{2} /$ Petroleum Ether): 0.40 . Mp: $82-83^{\circ} \mathrm{C}$. IR v ( $\left.\mathrm{cm}^{-1}\right): 3001$, 2956, 2930, 2837, 2241, 1605, 1505, 1462, 1246, 1180, 1030, 906, 806, 728. ${ }^{1} \mathbf{H}$ NMR (360 MHz, CDCl $): \delta 7.84$ $(\mathrm{d}, J=9.0 \mathrm{~Hz}, 2 \mathrm{H}), 7.18(\mathrm{~d}, J=9.0 \mathrm{~Hz}, 2 \mathrm{H}), 6.93-6.75(\mathrm{~m}, 6 \mathrm{H}), 6.68-6.57(\mathrm{~m}, 4 \mathrm{H}), 6.48(\mathrm{~d}, J=9.0 \mathrm{~Hz}, 2 \mathrm{H}), 4.28$ (dd, $J=12.0,6.6 \mathrm{~Hz}, 1 \mathrm{H}), 3.82(\mathrm{~s}, 3 \mathrm{H}), 3.74(\mathrm{~s}, 3 \mathrm{H}), 3.73(\mathrm{~s}, 3 \mathrm{H}), 3.72(\mathrm{~s}, 3 \mathrm{H}), 3.33(\mathrm{dd}, J=10.0,6.6 \mathrm{~Hz}, 1 \mathrm{H}), 2.82(\mathrm{dd}, J$ $=12.0,10.0 \mathrm{~Hz}, 1 \mathrm{H}) .{ }^{13} \mathrm{C} \mathrm{NMR}\left(63 \mathrm{MHz}, \mathrm{CDCl}_{3}\right): \delta 158.9$ (C), 158.7 (C), 158.1 (C), 157.7 (C), 138.8 (2C), 136.2 (C), $133.8(2 \mathrm{CH}), 132.2(2 \mathrm{CH}), 131.4(2 \mathrm{CH}), 131.2(2 \mathrm{CH}), 130.0(\mathrm{C}), 118.6(\mathrm{C}), 113.0(2 \mathrm{CH}), 112.6(2 \mathrm{CH}), 112.3(2 \mathrm{CH})$, $111.8(2 \mathrm{CH}), 72.1(\mathrm{C}), 65.9$ (C), $55.4\left(2 \mathrm{CH}_{3}\right), 55.3\left(2 \mathrm{CH}_{3}\right), 42.3(\mathrm{CH}), 30.3\left(\mathrm{CH}_{2}\right)$. HRMS (ESI-TOF) $\mathrm{m} / \mathrm{z}:[\mathrm{M}+\mathrm{Na}]^{+}$ Calcd for $\mathrm{C}_{33} \mathrm{H}_{31} \mathrm{NO}_{4} \mathrm{SNa}$ 560.1866. Found 560.1860. 
4,4,5,5-Tetrakis(4-fluorophenyl)tetrahydrothiophene-3-carbonitrile 4e

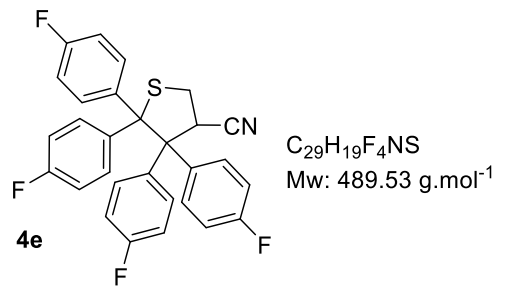

General procedure A was applied to 4,4'-difluorobenzophenone $\mathbf{V}$ (1.047 g, 4.8 $\mathrm{mmol})$ and acrylonitrile $2(7.27 \mathrm{~mL}, 110.4 \mathrm{mmol})$ in cyclohexane $(200 \mathrm{~mL})$. Flash chromatography (01:99 EtOAc/Petroleum ether to 10:90 EtOAc/Petroleum ether) gave product $4 \mathrm{e}$ as a white powder (568 mg, 48\%).

$R_{f}$ (06:94 EtOAc/Petroleum ether): 0.28 . Mp: $229-230^{\circ} \mathrm{C}$. IR v $\left(\mathrm{cm}^{-1}\right): 3080$, 2937, 2242, 1603, 1504, 1229, 1162, 1013, 822, 764, 747. ${ }^{1} \mathbf{H}$ NMR (360 MHz, CDCl $): \delta 7.88$ (dd, J = 8.2, $5.3 \mathrm{~Hz}$, $2 \mathrm{H}), 7.23-7.15(\mathrm{~m}, 2 \mathrm{H}), 7.05-6.78(\mathrm{~m}, 10 \mathrm{H}), 6.68(\mathrm{dd}, J=9.1,8.2 \mathrm{~Hz}, 2 \mathrm{H}), 4.28(\mathrm{dd}, J=12.0,6.6 \mathrm{~Hz}, 1 \mathrm{H}), 3.41$ (dd, $J=10.3,6.6 \mathrm{~Hz}, 1 \mathrm{H}), 2.85$ (dd, $J=12.0,10.3 \mathrm{~Hz}, 1 \mathrm{H}) .{ }^{13} \mathrm{C}$ NMR $\left(63 \mathrm{MHz}, \mathrm{CDCl}_{3}\right): \delta 162.5(\mathrm{~d}, J=249.7 \mathrm{~Hz}, \mathrm{C}), 162.1$ (d, J = 250.1 Hz, C), 161.6 (d, J = 249.1 Hz, C), 161.2 (d, J = 248.7 Hz, C), 141.7 (d, J = 2.6 Hz, C), 139.2 (d, J = 3.2 $\mathrm{Hz}, \mathrm{C}), 134.7$ (d, J = 3.0 Hz, C), 134.3 (d, J = 7.7 Hz, 2CH), 132.9 (d, J = 3.1 Hz, C), 132.6 (d, J = 8.0 Hz, 2CH), 131.8 (d, J = 7.7 Hz, 2CH), 131.5 (d, J = 7.7 Hz, 2CH), $117.6(\mathrm{~s}, \mathrm{C}), 115.1$ (d, J = 21.3 Hz, 2CH), 114.5 (d, J = 21.4 Hz, 2CH), $114.3(\mathrm{~d}, J=21.0 \mathrm{~Hz}, 2 \mathrm{CH}$ ), 113.7 (d, $J=21.4 \mathrm{~Hz}, 2 \mathrm{CH}), 71.9(\mathrm{~s}, \mathrm{C}), 66.0$ (s, C), $42.2(\mathrm{~s}, \mathrm{CH}), 30.3\left(\mathrm{~s}, \mathrm{CH}_{2}\right) .{ }^{19} \mathrm{~F} \mathrm{NMR}$ (235 MHz, $\mathrm{CDCl}_{3}$ ): $\delta-113.3,-113.4,-114.6,-115.2$. HRMS (ESI-TOF) $\mathrm{m} / \mathrm{z}$ : $[\mathrm{M}+\mathrm{Na}]^{+}$Calcd for $\mathrm{C}_{29} \mathrm{H}_{19} \mathrm{~F}_{4} \mathrm{NSNa}$ 512.1067. Found 512.1044.

\subsection{Preparation of thietanes 3a-c:}

\section{2,2-Diphenylthietane-3-carbonitrile 3a}

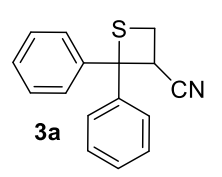

General procedure B was applied to benzophenone I (155 mg, $0.85 \mathrm{mmol})$ and acrylonitrile 2 (1.29 mL, $19.55 \mathrm{mmol}, 23$ equiv.) in cyclohexane $(5.2 \mathrm{~mL})$ for 1.5 h. Flash chromatography (02:98 EtOAc/Petroleum ether to 10:90 EtOAc/Petroleum ether) gave product $3 a$ as a white powder (91 $\mathrm{mg}, 43 \%)$.

General procedure B was applied to benzophenone I (1,82 g, $10.0 \mathrm{mmol})$ and acrylonitrile 2 (14.8 $\mathrm{mL}, 230.0$ $\mathrm{mmol}, 23$ equiv.) in cyclohexane $(22 \mathrm{~mL})$ for $10 \mathrm{~h}$. Flash chromatography (02:98 EtOAc/Petroleum ether to 5:95 EtOAc/Petroleum ether) gave product 3a as a white powder (1,0 g, 41\%).

$\mathbf{R}_{\mathbf{f}}$ (10:90 EtOAc/Petroleum ether): 0.45. Mp: 83-84 ${ }^{\circ} \mathrm{C}$. IR v (cm $\left.{ }^{-1}\right): 3054,2949,2239,1597,1489,1441,1315$, 1246, 1079, 1029, 747, 703. ${ }^{1} \mathrm{H}$ NMR (360 MHz, CDCl $)$ : $\delta$ 7.75-7.69 (m, 2H), 7.47-7.19 (m, 8H), 5.01 (app.t, J = 9.0 $\mathrm{Hz}, 1 \mathrm{H}$ ), 3.57 (app.t, $J=9.1 \mathrm{~Hz}, 1 \mathrm{H}$ ), 3.31 (app.t, $J=8.8 \mathrm{~Hz}, 1 \mathrm{H}) .{ }^{13} \mathrm{C} \mathrm{NMR} \mathrm{(90} \mathrm{MHz,} \mathrm{CDCl} 3$ ): $\delta 146.1$ (C), 140.5 (C), 128.7 (2CH), $128.6(2 \mathrm{CH}), 128.2(3 \mathrm{CH}), 127.5(\mathrm{CH}), 125.2(2 \mathrm{CH}), 117.7(\mathrm{C}), 60.9(\mathrm{C}), 39.5(\mathrm{CH}), 24.3\left(\mathrm{CH}_{2}\right)$. HRMS (ESI-TOF) $\mathrm{m} / z$ : $[\mathrm{M}+\mathrm{Na}]^{+}$Calcd for $\mathrm{C}_{16} \mathrm{H}_{13} \mathrm{NSNa}$ 274.0661. Found 274.0657. Spectral data were identical to those described in the literature. ${ }^{\mathrm{S1}}$

S1 Shima, K.; Sazaki, A.; Nakabayashi, K. Ring-splitting reaction of 2,2-diarylthietane by electron-transfer photosensitization of 9,10-dicyanoanthracene. Bull. Chem. Soc. Jpn. 1992, 65, 1472-1474. 


\section{2,2-Di-p-tolylthietane-3-carbonitrile 3b}

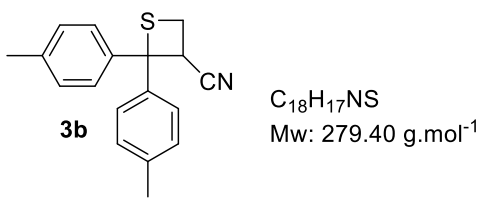

General procedure B was applied to 4,4'-dimethylbenzophenone II (241 mg, $1.15 \mathrm{mmol}$ ) and acrylonitrile $2(1.74 \mathrm{~mL}, 26.45 \mathrm{mmol}, 23$ equiv.) in cyclohexane

$(7.1 \mathrm{~mL})$ for $3.5 \mathrm{~h}$. Flash chromatography $(02: 98 \mathrm{EtOAc} /$ Petroleum ether to 10:90 EtOAc/Petroleum ether) gave product $\mathbf{3 b}$ as colorless oil (125 mg, 39\%).

$\mathbf{R}_{\mathbf{f}}$ (06:94 EtOAc/Petroleum ether): 0.42. IR v ( $\left.\mathrm{cm}^{-1}\right):$ 3023, 2920, 2861, 2243, 1609, 1507, 1447, 1185, 1125, 1021, 928, 809, 724. ${ }^{1} \mathrm{H}$ NMR $\left(250 \mathrm{MHz}, \mathrm{CDCl}_{3}\right): \delta 7.56$ (d, $\left.J=8.3 \mathrm{~Hz}, 2 \mathrm{H}\right), 7.24-7.12(\mathrm{~m}, 4 \mathrm{H}), 7.07(\mathrm{~d}, J=8.3 \mathrm{~Hz}$, 2H), 4.96 (app.t, $J=8.9 \mathrm{~Hz}, 1 \mathrm{H}$ ), 3.54 (app.t, $J=9.1 \mathrm{~Hz}, 1 \mathrm{H}$ ), 3.29 (app.t, $J=8.8 \mathrm{~Hz}, 1 \mathrm{H}), 2.35$ (s, 6H). ${ }^{13} \mathrm{C}$ NMR (90 MHz, $\left.\mathrm{CDCl}_{3}\right): \delta 143.5$ (C), 138.1 (C), 137.9 (C), 137.3 (C), $129.4(2 \mathrm{CH}), 129.3(2 \mathrm{CH}), 128.2(2 \mathrm{CH}), 125.2(2 \mathrm{CH}), 117.9$ (C), 60.9 (C), $39.7(\mathrm{CH}), 24.4\left(\mathrm{CH}_{2}\right), 21.2\left(2 \mathrm{CH}_{3}\right)$. HRMS (ESI-TOF) $\mathrm{m} / \mathrm{z}$ : $[\mathrm{M}+\mathrm{Na}]^{+}$Calcd for $\mathrm{C}_{18} \mathrm{H}_{17} \mathrm{NSNa} 302.0974$. Found 302.0970. Spectral data were identical to those described in the literature. ${ }^{\mathrm{S1}}$

\section{2,2-Bis(4-chlorophenyl)thietane-3-carbonitrile 3c}

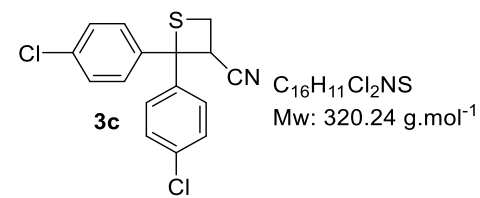

General procedure B was applied to 4,4'-dichlorobenzophenone III (226 mg, $0.85 \mathrm{mmol}$ ) and acrylonitrile 2 (1.29 $\mathrm{mL}, 19.55 \mathrm{mmol}, 23$ equiv.) in cyclohexane

$(5.2 \mathrm{~mL})$ for $4.5 \mathrm{~h}$. Flash chromatography (02:98 EtOAc/Petroleum ether to 10:90 EtOAc/Petroleum ether) gave product $3 c$ as colorless oil (115 mg, $42 \%)$.

$\mathbf{R}_{\mathbf{f}}$ (10:90 EtOAc/Petroleum ether): 0.33. IR v ( $\left.\mathrm{cm}^{-1}\right)$ : 3064, 2951, 2865, 2243, 1592, 1490, 1399, 1093, 1012, 906, 822, 727. ${ }^{1} \mathrm{H}$ NMR (300 MHz, $\left.\mathrm{CDCl}_{3}\right): \delta 7.61(\mathrm{~d}, J=8.7 \mathrm{~Hz}, 2 \mathrm{H}), 7.43-7.31(\mathrm{~m}, 4 \mathrm{H}), 7.10(\mathrm{~d}, J=8.7 \mathrm{~Hz}, 2 \mathrm{H}), 4.94$ (app.t, $J=9.0 \mathrm{~Hz}, 1 \mathrm{H}$ ), 3.53 (app.t, $J=9.1 \mathrm{~Hz}, 1 \mathrm{H}$ ), 3.32 (app.t, $J=8.9 \mathrm{~Hz}, 1 \mathrm{H}) .{ }^{13} \mathrm{C} \mathbf{N M R}\left(90 \mathrm{MHz}, \mathrm{CDCl}_{3}\right): \delta 144.1$ (C), 138.6 (C), 134.5 (C), 133.6 (C), $129.7(2 \mathrm{CH}), 129.0(2 \mathrm{CH}), 128.8(2 \mathrm{CH}), 126.7(2 \mathrm{CH}), 117.2(\mathrm{C}), 59.7(\mathrm{C}), 39.3$ (CH), $24.1\left(\mathrm{CH}_{2}\right)$. HRMS (ESI-TOF) $\mathrm{m} / z$ : [M + Na] $]^{+}$Calcd for $\mathrm{C}_{16} \mathrm{H}_{11}{ }^{35} \mathrm{Cl}_{2} \mathrm{NSNa}$ 341.9887. Found 341.9889.

\subsection{Preparation of "hetero" tetra-aryl tetrahydrothiophenes 4 from thietanes 3a-c:}

\section{4,4,5,5-Tetraphenyltetrahydrothiophene-3-carbonitrile 4a}

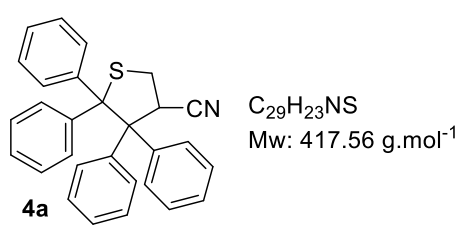

Spectral data were identical to those described above.
General procedure $\mathbf{C}$ was applied to benzophenone I ( $58 \mathrm{mg}, 0.32 \mathrm{mmol}, 2$ equiv.) and thietane 3a (39 mg, $0.16 \mathrm{mmol})$ in cyclohexane $(200 \mathrm{~mL})$. Flash chromatography (04:96 EtOAc/Petroleum ether to 06:94 EtOAc/Petroleum ether) gave product $4 \mathrm{a}$ as white powder (50 $\mathrm{mg}, 76 \%$ ). 


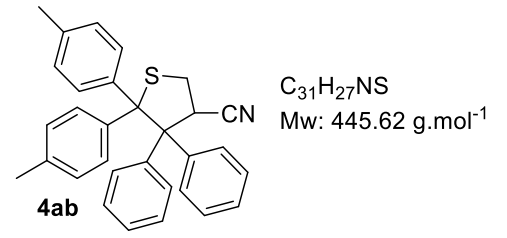

General procedure C was applied to 4,4'-dimethyl benzophenone II (101 mg, $0.48 \mathrm{mmol}, 2$ equiv.) and thietane $3 \mathbf{3}(60 \mathrm{mg}, 0.24 \mathrm{mmol})$ in cyclohexane (200 $\mathrm{mL})$. Flash chromatography (02:98 EtOAc/Petroleum ether to 04:96 EtOAc/Petroleum ether) gave product $4 \mathrm{ab}$ as white powder (86 mg, 81\%).

$\mathbf{R}_{\mathrm{f}}\left(\mathbf{1 0 : 9 0}\right.$ EtOAc/Petroleum Ether): 0.58. Mp: $126-127^{\circ} \mathrm{C} . \mathrm{IR} \mathbf{v}\left(\mathrm{cm}^{-1}\right): 3001$, 2920, 2857, 2240, 1499, 1443, 1192, 1019, 1038, 752, 735, 711. ${ }^{1} \mathrm{H}$ NMR $(250$ MHz, CDCl $): \delta 7.94(d, J=7.2 \mathrm{~Hz}$, $2 \mathrm{H}), 7.42-6.66(\mathrm{~m}, 16 \mathrm{H}), 4.43(\mathrm{dd}, J=12.0,6.6 \mathrm{~Hz}, 1 \mathrm{H}), 3.38(\mathrm{dd}, J=10.0,6.6 \mathrm{~Hz}, 1 \mathrm{H}), 2.87(\mathrm{dd}, J=12.0,10.0 \mathrm{~Hz}$, 1H), 2.26 (s, 3H), $2.21(\mathrm{~s}, 3 \mathrm{H}) .{ }^{13} \mathrm{C}$ NMR (63 MHz, CDCl 3 ): $\delta 143.5$ (C), 140.9 (C), 139.5 (C), 138.2 (C), 136.2 (C), $136.1(\mathrm{C}), 132.6(2 \mathrm{CH}), 130.9(2 \mathrm{CH}), 130.3(2 \mathrm{CH}), 130.1(2 \mathrm{CH}), 127.9(3 \mathrm{CH}), 127.6(2 \mathrm{CH}), 127.4(\mathrm{CH}), 127.2(2 \mathrm{CH})$, $127.1(2 \mathrm{CH}), 118.4(\mathrm{C}), 72.9$ (C), 66.7 (C), $41.8(\mathrm{CH}), 30.5\left(\mathrm{CH}_{2}\right), 21.0\left(\mathrm{CH}_{3}\right), 20.8\left(\mathrm{CH}_{3}\right)$. HRMS (ESI-TOF) $\mathrm{m} / \mathrm{z}:[\mathrm{M}+$ $\mathrm{Na}]^{+}$Calcd for $\mathrm{C}_{31} \mathrm{H}_{27} \mathrm{NSNa}$ 468.1762. Found 468.1736.

\section{5,5-Bis(4-chlorophenyl)-4,4-diphenyltetrahydrothiophene-3-carbonitrile 4ac}

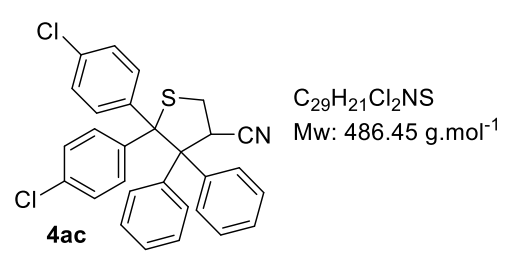

General procedure C was applied to 4,4'-dichloro benzophenone III (181 mg, $0.72 \mathrm{mmol}, 2$ equiv.) and thietane $3 \mathbf{a}(90 \mathrm{mg}, 0.36 \mathrm{mmol})$ in cyclohexane (200 $\mathrm{mL})$. Flash chromatography (02:98 EtOAc/Petroleum ether to 04:96 EtOAc/Petroleum ether) gave product 4ac as white powder (157 mg, 90\%).

$\mathbf{R}_{\mathrm{f}}$ (04:96, Ethyl acetate/Petroleum Ether): 0.18 . $\mathbf{M p :} 230-231^{\circ} \mathrm{C}$. IR v $\left(\mathrm{cm}^{-1}\right)$ : $3066,2936,2238,1589,1487,1443,1397,1263,1094,1012,908,803,734,714,700 .{ }^{1}{ }^{H}$ NMR (360 MHz, CDCl $)$ : $\delta 7.88(\mathrm{~d}, J=7.7 \mathrm{~Hz}, 2 \mathrm{H}), 7.43-6.74(\mathrm{~m}, 16 \mathrm{H}), 4.31(\mathrm{dd}, J=12.0,6.5 \mathrm{~Hz}, 1 \mathrm{H}), 3.39(\mathrm{dd}, J=10.3,6.5 \mathrm{~Hz}, 1 \mathrm{H}), 2.90$ (dd, $J=12.0,10.3 \mathrm{~Hz}, 1 \mathrm{H}) .{ }^{13} \mathrm{C}$ NMR (100 MHz, CDCl $)$ : $\delta 144.5$ (C), 142.0 (C), 138.8 (C), 137.3 (C), 133.1 (C), 132.7 (C), $132.4(2 \mathrm{CH}), 132.2(2 \mathrm{CH}), 131.7(2 \mathrm{CH}), 129.8(2 \mathrm{CH}), 128.4(\mathrm{CH}), 128.1(2 \mathrm{CH}), 128.0(\mathrm{CH}), 127.6(2 \mathrm{CH}), 127.4$ (2CH), $126.8(2 \mathrm{CH}), 117.8$ (C), 72.1 (C), 66.8 (C), 41.9 (CH), $30.6\left(\mathrm{CH}_{2}\right)$. HRMS (ESI-TOF) m/z: [M + Na] $]^{+}$Calcd for $\mathrm{C}_{29} \mathrm{H}_{21}{ }^{35} \mathrm{Cl}_{2} \mathrm{NSNa} 508.0669$ Found 508.0639.

\section{5,5-Bis(4-methoxyphenyl)-4,4-diphenyltetrahydrothiophene-3-carbonitrile 4ad}

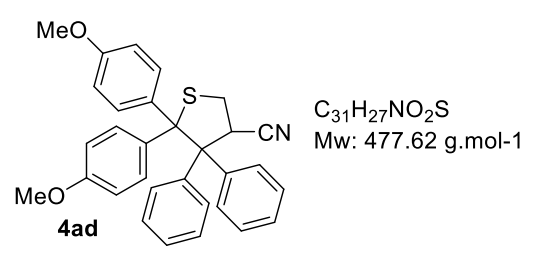

General procedure C was applied to 4,4'-dimethoxybenzophenone IV (111 $\mathrm{mg}, 0.46 \mathrm{mmol}, 2$ equiv.) and thietane $3 \mathrm{a}(58 \mathrm{mg}, 0.23 \mathrm{mmol})$ in cyclohexane $(200 \mathrm{~mL})$. Flash chromatography (02:98 EtOAc/Petroleum ether to 10:90 EtOAc/Petroleum ether) gave product 4ad as white powder (73 mg, 66\%).

$\mathbf{R}_{\mathrm{f}}$ (10:90 EtOAc/Petroleum Ether): 0.19. Mp: $125-127^{\circ} \mathrm{C}$. IR v $\left(\mathrm{cm}^{-1}\right): 2955$, 2931, 2835, 2237, 1604, 1505, 1442, 1299, 1247, 1177, 1030, 815, 737, 710. ${ }^{1} \mathbf{H}$ NMR (250 MHz, CDCl 3 ): $\delta 7.94$ $(\mathrm{d}, J=7.2 \mathrm{~Hz}, 2 \mathrm{H}), 7.40-6.93(\mathrm{~m}, 10 \mathrm{H}), 6.81(\mathrm{~d}, J=8.6 \mathrm{~Hz}, 2 \mathrm{H}), 6.65(\mathrm{~d}, J=8.9 \mathrm{~Hz}, 2 \mathrm{H}), 6.44(\mathrm{~d}, J=8.9 \mathrm{~Hz}, 2 \mathrm{H}), 4.39$ (dd, $J=12.0,6.6 \mathrm{~Hz}, 1 \mathrm{H}$ ), $3.74(\mathrm{~s}, 3 \mathrm{H}), 3.72(\mathrm{~s}, 3 \mathrm{H}), 3.37$ (dd, $J=10.2,6.6 \mathrm{~Hz}, 1 \mathrm{H}), 2.87(\mathrm{dd}, J=12.0,10.2 \mathrm{~Hz}, 1 \mathrm{H}$ ). 
${ }^{13} \mathrm{C}$ NMR (90 MHz, CDCl $): \delta 158.1$ (C), 157.7 (C), 139.5 (C), 138.7 (C), 138.1 (C), 136.0 (C), 132.6 (2CH), 132.1 (2CH), $131.5(2 \mathrm{CH}), 130.0(2 \mathrm{CH}), 128.0(\mathrm{CH}), 127.7(2 \mathrm{CH}), 127.5(\mathrm{CH}), 127.1(2 \mathrm{CH}), 118.4(\mathrm{C}), 112.5(2 \mathrm{CH}), 111.8$ (2CH), $72.3(\mathrm{C}), 66.9$ (C), $55.4\left(\mathrm{CH}_{3}\right), 55.2\left(\mathrm{CH}_{3}\right), 41.8(\mathrm{CH}), 30.5\left(\mathrm{CH}_{2}\right)$. HRMS (ESI-TOF) $\mathrm{m} / \mathrm{z}$ : [M + Na] ${ }^{+}$Calcd for $\mathrm{C}_{31} \mathrm{H}_{27} \mathrm{NO}_{2} \mathrm{SNa}$ 500.1660. Found 500.1660.

\section{5,5-Bis(4-fluorophenyl)-4,4-diphenyltetrahydrothiophene-3-carbonitrile 4ae}

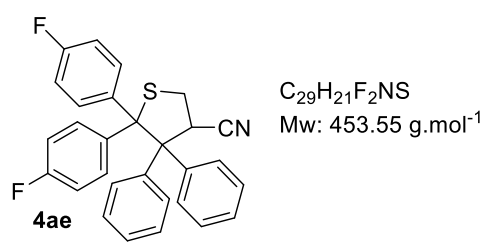

General procedure $\mathbf{C}$ was applied to 4,4'-difluoro benzophenone V (101 mg, $0.46 \mathrm{mmol}, 2$ equiv.) and thietane $3 \mathrm{a}(58 \mathrm{mg}, 0.23 \mathrm{mmol})$ in cyclohexane (200 $\mathrm{mL})$. Flash chromatography (02:98 EtOAc/Petroleum ether to 05:95 EtOAc/Petroleum ether) gave product 4 ae as white powder (72 mg, 69\%).

$R_{\mathrm{f}}\left(10: 90\right.$ EtOAc/Petroleum ether): 0.42. Mp: $233-234{ }^{\circ} \mathrm{C}$. IR v $\left(\mathrm{cm}^{-1}\right): 3064$,

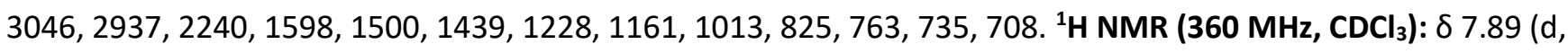
$J=7.6 \mathrm{~Hz}, 2 \mathrm{H}), 7.42-6.76(\mathrm{~m}, 14 \mathrm{H}), 6.62(\mathrm{t}, J=8.6 \mathrm{~Hz}, 2 \mathrm{H}), 4.34(\mathrm{dd}, J=12.0,6.6 \mathrm{~Hz}, 1 \mathrm{H}), 3.39$ (dd, $J=10.4,6.6$ $\mathrm{Hz}, 1 \mathrm{H}), 2.91$ (dd, $J=12.0,10.4 \mathrm{~Hz}, 1 \mathrm{H}) .{ }^{13} \mathrm{C}$ NMR (63 MHz, CDCl $): \delta 161.5$ (d, J=248.7 Hz, C), 161.1 (d, $J=247.9$ $\mathrm{Hz}, \mathrm{C}), 142.0$ (s, C), 139.6 (s, C), 139.0 (s, C), 137.5 (s, C), 132.6 (d, J = 8.7 Hz, 2CH), 132.5 (s, 2CH), 132.0 (d, J = 7.6 $\mathrm{Hz}, 2 \mathrm{CH}), 129.8(\mathrm{~s}, 2 \mathrm{CH}), 128.3(\mathrm{~s}, \mathrm{CH}), 128.0(\mathrm{~s}, 2 \mathrm{CH}), 127.9(\mathrm{~s}, \mathrm{CH}), 127.3(2 \mathrm{CH}), 117.9(\mathrm{~s}, \mathrm{C}), 114.3(\mathrm{~d}, J=21.3$ $\mathrm{Hz}, 2 \mathrm{CH}), 113.4(\mathrm{~d}, J=21.4 \mathrm{~Hz}, 2 \mathrm{CH}), 72.0(\mathrm{~s}, \mathrm{C}), 66.9(\mathrm{~s}, \mathrm{C}), 41.9(\mathrm{~s}, \mathrm{CH}), 30.5\left(\mathrm{~s}, \mathrm{CH}_{2}\right) .{ }^{19} \mathrm{~F}$ NMR (235 MHz, CDCl$)$ : $\delta$-115.2, -116.0. HRMS (ESI-TOF) $\mathrm{m} / \mathrm{z}$ : $[\mathrm{M}+\mathrm{Na}]^{+}$Calcd for $\mathrm{C}_{29} \mathrm{H}_{21} \mathrm{~F}_{2} \mathrm{NSNa}$ 476.1260. Found 476.1292.

\section{5,5-Diphenyl-4,4-di-p-tolyltetrahydrothiophene-3-carbonitrile 4ba}

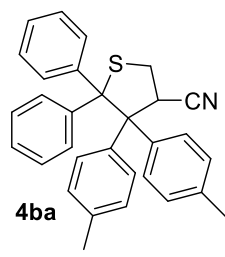

General procedure C was applied to benzophenone I (40 mg, $0.22 \mathrm{mmol}, 2$ equiv.) and thietane $3 \mathbf{b}(32 \mathrm{mg}, 0.11 \mathrm{mmol})$ in cyclohexane $(200 \mathrm{~mL})$. Flash $\mathrm{C}_{31} \mathrm{H}_{27} \mathrm{NS}$ chromatography (02:98 EtOAc/Petroleum ether to 10:90 EtOAc/Petroleum ether) gave product $\mathbf{4 b a}$ as white powder ( $43 \mathrm{mg}, 84 \%)$.

$\mathbf{R}_{\mathrm{f}}\left(\mathbf{1 0 : 9 0}\right.$ EtOAc/Petroleum Ether): 0.53. Mp: 220-221 ${ }^{\circ} \mathrm{C}$. IR v (cm $\left.{ }^{-1}\right): 3031,2922,2856,2238,1509,1487,1442$, 1293, 1187, 1022, 813, 747, 732, 701. ${ }^{1} \mathrm{H}$ NMR (360 MHz, CDCl $): \delta 7.79$ (d, J = 8.2 Hz, 2H), 7.36-6.75 (m, $\left.16 \mathrm{H}\right)$, $4.37(\mathrm{dd}, J=11.9,6.5 \mathrm{~Hz}, 1 \mathrm{H}), 3.36(\mathrm{dd}, J=10.2,6.5 \mathrm{~Hz}, 1 \mathrm{H}), 2.86(\mathrm{dd}, J=11.9,10.2 \mathrm{~Hz}, 1 \mathrm{H}), 2.36(\mathrm{~s}, 3 \mathrm{H}), 2.24(\mathrm{~s}$, 3H). $\left.{ }^{13} \mathrm{C} \mathrm{NMR} \mathrm{(90} \mathrm{MHz,} \mathrm{CDCl}\right)$ : $\delta 146.3$ (C), 144.1 (C), 137.6 (C), 137.2 (C), 136.2 (C), 134.9 (C), 132.5 (2CH), 131.1 (2CH), $130.4(2 \mathrm{CH}), 130.0(2 \mathrm{CH}), 128.3(2 \mathrm{CH}), 127.8(2 \mathrm{CH}), 127.3(2 \mathrm{CH}), 126.52(2 \mathrm{CH}), 126.49(\mathrm{CH}), 126.45(\mathrm{CH})$, 118.5 (C), 73.0 (C), 66.3 (C), $42.0(\mathrm{CH}), 30.3\left(\mathrm{CH}_{2}\right), 21.1\left(\mathrm{CH}_{3}\right), 20.9\left(\mathrm{CH}_{3}\right)$. HRMS (ESI-TOF) m/z: [M + Na] $]^{+}$Calcd for $\mathrm{C}_{31} \mathrm{H}_{27} \mathrm{NSNa}$ 468.1756. Found 468.1731. 
4,4-Bis(4-chlorophenyl)-5,5-diphenyltetrahydrothiophene-3-carbonitrile 4ca

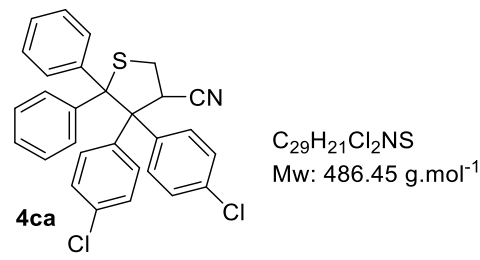

General procedure C was applied to benzophenone I $(55 \mathrm{mg}, 0.30 \mathrm{mmol}, 2$ equiv.) and thietane $3 c(48 \mathrm{mg}, 0.15 \mathrm{mmol})$ in cyclohexane $(200 \mathrm{~mL})$. Flash chromatography (02:98 EtOAc/Petroleum ether to 10:90 EtOAc/Petroleum ether) gave product 4ca as white powder (51 mg, 70\%).

$\mathbf{R}_{\mathbf{f}}\left(\mathbf{1 0 : 9 0}\right.$ EtOAc/Petroleum Ether): 0.42. Mp: 232-233 ${ }^{\circ} \mathrm{C}$. IR v ( $\left.\mathrm{cm}^{-1}\right): 3060,2922,2852,2239,1591,1492,1442$, 1404, 1259, 1100, 1014, 819, 788, 752, 735, 700. ${ }^{1} \mathbf{H}$ NMR (360 MHz, CDCl $): \delta 7.86$ (d, J = 8.7 Hz, 2H), 7.33-6.78 $(\mathrm{m}, 16 \mathrm{H}), 4.37(\mathrm{dd}, J=12.0,6.6 \mathrm{~Hz}, 1 \mathrm{H}), 3.41(\mathrm{dd}, J=10.3,6.6 \mathrm{~Hz}, 1 \mathrm{H}), 2.83(\mathrm{dd}, J=12.0,10.3 \mathrm{~Hz}, 1 \mathrm{H}) .{ }^{13} \mathrm{C}$ NMR (90 MHz, CDCl $)$ : $\delta 145.6$ (C), 143.2 (C), 137.5 (C), 135.9 (C), $134.4(2 \mathrm{C}), 133.9(2 \mathrm{CH}), 131.3(2 \mathrm{CH}), 131.0(2 \mathrm{CH})$, $130.1(2 \mathrm{CH}), 128.0(2 \mathrm{CH}), 127.6(2 \mathrm{CH}), 127.5(2 \mathrm{CH}), 127.0(\mathrm{CH}), 126.9(3 \mathrm{CH}), 117.8(\mathrm{C}), 72.9(\mathrm{C}), 66.0(\mathrm{C}), 41.9$ (CH), $30.2\left(\mathrm{CH}_{2}\right)$. HRMS (ESI-TOF) m/z: [M + Na] $]^{+}$Calcd for $\mathrm{C}_{29} \mathrm{H}_{21}{ }^{35} \mathrm{Cl}_{2} \mathrm{NSNa}$ 508.0670. Found 508.0653.

\section{4,4-Bis(4-chlorophenyl)-5,5-di-p-tolyltetrahydrothiophene-3-carbonitrile 4cb}

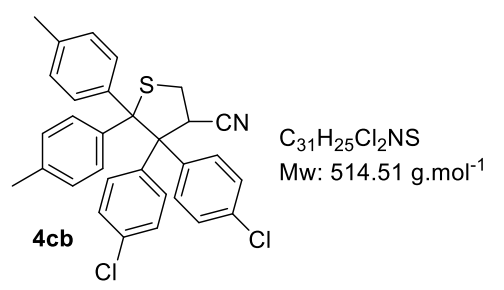

General procedure C was applied to 4,4'-dimethylbenzophenone II (100 mg, $0.46 \mathrm{mmol}, 2$ equiv.) and thietane $3 \mathrm{c}(73 \mathrm{mg}, 0.23 \mathrm{mmol})$ in cyclohexane (200 $\mathrm{mL})$. Flash chromatography (02:98 EtOAc/Petroleum ether to 07:93 EtOAc/Petroleum ether) gave product $4 \mathrm{cb}$ as white powder (101 mg, 86\%).

$\mathbf{R}_{\mathrm{f}}\left(\mathbf{1 0 : 9 0}\right.$ EtOAc/Petroleum ether): $0.56 . \mathrm{Mp:} 231-232^{\circ} \mathrm{C}$. IR $\mathbf{v}\left(\mathrm{cm}^{-1}\right): 3018$, 2933, 2246, 1592, 1493, 1400, 1098, 1009, 805, 772. ${ }^{1} \mathrm{H}$ NMR (360 MHz, CDCl $): \delta 7.87$ (d, J = 8.6 Hz, 2H), 7.27 (d, J = 8.6 Hz, 2H), 7.12-7.01 (m, 4H), 6.97-6.71 (m, 8H), $4.36(\mathrm{dd}, J=11.9,6.6 \mathrm{~Hz}, 1 \mathrm{H}), 3.39(\mathrm{dd}, J=10.2,6.6 \mathrm{~Hz}$, 1H), $2.81(\mathrm{dd}, J=11.9,10.2 \mathrm{~Hz}, 1 \mathrm{H}), 2.26(\mathrm{~s}, 3 \mathrm{H}), 2.24(\mathrm{~s}, 3 \mathrm{H}) .{ }^{13} \mathrm{C}$ NMR (90 MHz, CDCl 3 ): $\delta 142.8(\mathrm{C}), 140.2(\mathrm{C})$, 137.6 (C), 136.7 (C), 136.5 (C), 136.1 (C), 134.2 (C), $133.9(2 \mathrm{CH}), 133.7(\mathrm{C}), 131.4(2 \mathrm{CH}), 130.9(2 \mathrm{CH}), 129.9(2 \mathrm{CH})$, $128.2(2 \mathrm{CH}), 127.8(2 \mathrm{CH}), 127.5(2 \mathrm{CH}), 127.3(2 \mathrm{CH}), 117.9(\mathrm{C}), 72.5(\mathrm{C}), 65.9(\mathrm{C}), 41.8(\mathrm{CH}), 30.1\left(\mathrm{CH}_{2}\right), 21.0\left(\mathrm{CH}_{3}\right)$, $20.9\left(\mathrm{CH}_{3}\right)$. HRMS (ESI-TOF) $\mathrm{m} / z$ : $[\mathrm{M}+\mathrm{Na}]^{+}$Calcd for $\mathrm{C}_{31} \mathrm{H}_{25}{ }^{35} \mathrm{Cl}_{2} \mathrm{NSNa} 536.0982$ Found 536.0953.

\section{4,4-Diphenyl-5,5-bis(3-(trifluoromethyl)phenyl)tetrahydrothiophene-3-carbonitrile 4af}

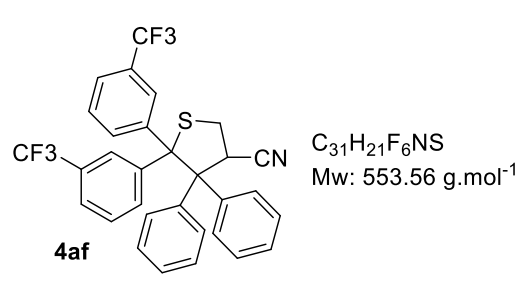
General procedure $\mathbf{C}$ was applied to 3,3'-Bis(trifluoromethyl) benzophenone VI (285 mg, $0.90 \mathrm{mmol}, 3$ equiv.) and thietane $3 \mathrm{a}$ (75 mg, $0.30 \mathrm{mmol}$ ) for cyclohexane (200 mL). Flash chromatography (02:98 EtOAc/Petroleum ether to $10: 90 \mathrm{EtOAc} /$ Petroleum ether) gave product 4 af as white powder (63 mg, $38 \%)$.

$\mathbf{R}_{\mathbf{f}}$ (08:92 EtOAc/Petroleum Ether): 0.28. Mp: 68-70 ${ }^{\circ} \mathrm{C}$. IR v (cm $\left.{ }^{-1}\right): 2961,2925,2240,1593,1488,1444,1326$, 1261, 1115, 1075, 1013, 793, 707. ${ }^{1} \mathrm{H}$ NMR (360 MHz, CDCl $): \delta 7.87$ (d, J = 7.7 Hz, 2H), 7.62-6.79 (m, 16H), 4.29 (dd, $J=12.1,6.5 \mathrm{~Hz}, 1 \mathrm{H}$ ), 3.48 (dd, $J=10.3,6.5 \mathrm{~Hz}, 1 \mathrm{H}$ ), 3.00 (dd, $J=12.1,10.3 \mathrm{~Hz}, 1 \mathrm{H}) .{ }^{13} \mathrm{C} \mathrm{NMR}\left(75 \mathrm{MHz}, \mathrm{CDCl}_{3}\right)$ : $\delta 146.6$ (C), 144.2 (C), 138.4 (C), 136.9 (C), $134.0(\mathrm{CH}), 133.8(\mathrm{CH}), 132.2(2 \mathrm{CH}), 130.0(\mathrm{C}), 129.6(2 \mathrm{CH}), 129.3(\mathrm{C})$, 
$128.6(\mathrm{CH}), 128.3(3 \mathrm{CH}), 128.2(\mathrm{CH}), 127.5(3 \mathrm{CH}), 127.4(\mathrm{CH}), 127.0(\mathrm{CH}), 123.92(\mathrm{CH}), 123.88(\mathrm{CH}), 123.84(\mathrm{~d}, \mathrm{~J}=$ $272.2 \mathrm{~Hz}, \mathrm{C}), 123.80$ (d, J = 272.7 Hz, C), 117.5 (C), 72.4 (C), 67.0 (C), $41.9(\mathrm{CH}), 30.6\left(\mathrm{CH}_{2}\right) .{ }^{19} \mathrm{~F} \mathrm{NMR}(235 \mathrm{MHz}$, $\mathrm{CDCl}_{3}$ ): $\delta$-62.4, -62.9. HRMS (ESI-TOF) $\mathrm{m} / \mathrm{z}$ : $[\mathrm{M}+\mathrm{Na}]^{+}$Calcd for $\mathrm{C}_{31} \mathrm{H}_{21} \mathrm{~F}_{6} \mathrm{NSNa}$ 576.1163. Found 576.1191.

\section{4,4,5-triphenyl-5-(p-tolyl)tetrahydrothiophene-3-carbonitrile 4ag}

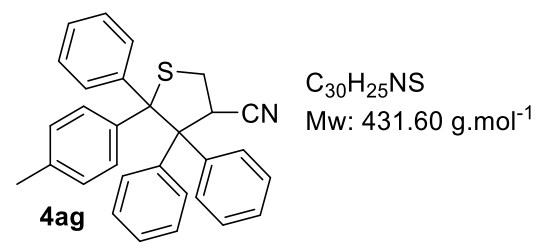

General procedure C was applied to 4-methyl benzophenone VII (106 mg, $0.5 \mathrm{mmol}, 2$ equiv.) and thietane $3 \mathrm{a}$ (63 $\mathrm{mg}, 0.25 \mathrm{mmol}$ ) in cyclohexane (200 $\mathrm{mL})$. Flash chromatography (02:98 EtOAc/Petroleum ether to 05:96 EtOAc/Petroleum ether) of the crude mixture of diastereoisomers 4 ag (d.r. $50 / 50$ by ${ }^{1} \mathrm{H}$ NMR signal integration of the crude reaction mixture) gave diastereoisomer 4ag1 (33 mg, 31\%) and diastereoisomer 4ag2 (39 mg, 36\%) as pale yellow powders.

4ag1 (spectal data were deduced from a sample containing 4ag1 and $4 \mathrm{ag} 2): \mathbf{R}_{\mathrm{f}}$ (5:95 EtOAc/Petroleum Ether): 0.38. Mp: not determined (obtained as a mixture). IR v (cm $\left.{ }^{-1}\right): 3056,2922,2859,2241,1495,1443,1191,1087$, 1031, 799, 735, 701. ${ }^{1} \mathrm{H}$ NMR (400 MHz, $\left.\mathrm{CDCl}_{3}\right): \delta 7.92(\mathrm{~d}, \mathrm{~J}=7.8 \mathrm{~Hz}, 2 \mathrm{H}), 7.36-7.27(\mathrm{~m}, 4 \mathrm{H}), 7.18-7.08(\mathrm{~m}, 4 \mathrm{H})$, 7.07-6.95 (m, 4H), 6.94-6.89 (m, 5H), $4.43(\mathrm{dd}, J=12.1,6.4 \mathrm{~Hz}, 1 \mathrm{H}), 3.38(\mathrm{dd}, J=10.2,6.4 \mathrm{~Hz}, 1 \mathrm{H}), 2.86(\mathrm{dd}, J=$ 12.1, 10.2 Hz, 1H), 2.21 (s, 3H). ${ }^{13} \mathrm{C}$ NMR (100 MHz, CDCl $)$ : $\delta 144.1$ (C), 143.3 (C), 139.4 (C), 138.0 (C), 136.3 (C), $132.6(2 \mathrm{CH}), 131.0(2 \mathrm{CH}), 130.4(2 \mathrm{CH}), 130.2(2 \mathrm{CH}), 128.0(\mathrm{CH}), 127.7(2 \mathrm{CH}), 127.5(\mathrm{CH}), 127.30(2 \mathrm{CH}), 127.27$ (2CH), $127.1(2 \mathrm{CH}), 126.5(\mathrm{CH}), 118.4$ (C), 73.0 (C), 66.8 (C), $41.9(\mathrm{CH}), 30.4\left(\mathrm{CH}_{2}\right), 20.9\left(\mathrm{CH}_{3}\right)$. HRMS (ESI-TOF) $\mathrm{m} / \mathrm{z}:[\mathrm{M}+\mathrm{Na}]^{+}$Calcd for $\mathrm{C}_{30} \mathrm{H}_{25} \mathrm{NSNa}$ 454.1600. Found 454.1578.

4ag2: $\mathbf{R}_{\mathbf{f}}$ (5:95 EtOAc/Petroleum Ether): 0.37. Mp: 182-184 ${ }^{\circ} \mathrm{C} . \mathbf{I R} \mathbf{v}\left(\mathrm{cm}^{-1}\right): 3046,2922,2855,2243,1488,1441$, 1191, 1084, 1036, 797, 767, 701. ${ }^{1} \mathrm{H}$ NMR (400 MHz, $\left.\mathrm{CDCl}_{3}\right): \delta 7.94(\mathrm{~d}, J=7.7 \mathrm{~Hz}, 2 \mathrm{H})$, 7.36-7.27 (m, 4H), 7.18$7.08(\mathrm{~m}, 4 \mathrm{H}), 7.07-6.95(\mathrm{~m}, 4 \mathrm{H}), 6.94-6.89(\mathrm{~m}, 5 \mathrm{H}), 4.42(\mathrm{dd}, J=12.0,6.5 \mathrm{~Hz}, 1 \mathrm{H}), 3.39(\mathrm{dd}, J=10.1,6.5 \mathrm{~Hz}, 1 \mathrm{H})$, $2.90(\mathrm{dd}, J=12.0,10.1 \mathrm{~Hz}, 1 \mathrm{H}), 2.26(\mathrm{~s}, 3 \mathrm{H}) .{ }^{13} \mathrm{C}$ NMR (100 MHz, CDCl 3 ): $\delta 146.4$ (C), 140.8 (C), 139.5 (C), 138.1 (C), $136.2(\mathrm{C}), 132.6(2 \mathrm{CH}), 131.0(2 \mathrm{CH}), 130.4(2 \mathrm{CH}), 130.0(2 \mathrm{CH}), 128.02(\mathrm{CH}), 127.98(2 \mathrm{CH}), 127.7(2 \mathrm{CH}), 127.5$ (CH), $127.1(2 \mathrm{CH}), 126.54(2 \mathrm{CH}), 126.49(\mathrm{CH}), 118.3(\mathrm{C}), 73.1(\mathrm{C}), 66.8(\mathrm{C}), 41.8(\mathrm{CH}), 30.5\left(\mathrm{CH}_{2}\right), 21.0\left(\mathrm{CH}_{3}\right)$. HRMS (ESI-TOF) $\mathrm{m} / \mathrm{z}$ : [M + Na] $]^{+}$Calcd for $\mathrm{C}_{30} \mathrm{H}_{25} \mathrm{NSNa}$ 454.1600. Found 454.1579.

\subsection{Procedures and analytical data for additional experiments:}

Photochemical reaction of thiobenzophenone and acrylonitrile in different experimental conditions in cyclohexane, benzene and toluene (Table 1, entries 1-13)
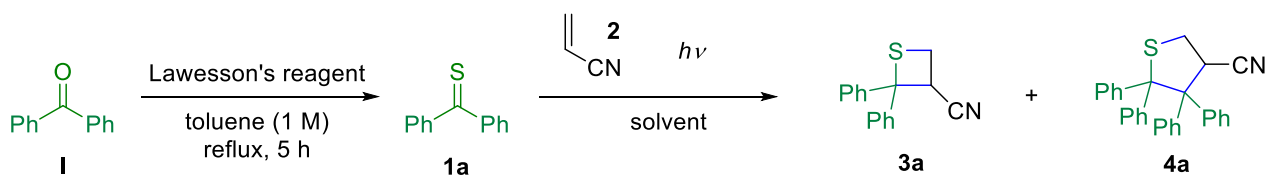
Entry 1: General procedure B was applied to benzophenone I (155 mg, $0.85 \mathrm{mmol})$ and acrylonitrile $2(1.29 \mathrm{~mL}$, $19.55 \mathrm{mmol}$ ) in cyclohexane $(5.2 \mathrm{~mL})$ for $1.5 \mathrm{~h}$ using $\mathrm{Hg}$ lamp. Flash chromatography (02:98 EtOAc/Petroleum ether to 10:90 EtOAc/Petroleum ether) gave product 3a as a white powder (91 mg, 43\%).

Entry 2: General procedure B was applied to benzophenone I (155 mg, $0.85 \mathrm{mmol}$ ) and acrylonitrile 2 (1.29 mL, $19.55 \mathrm{mmol}$ ) in cyclohexane $(2.6 \mathrm{~mL})$ for $1.5 \mathrm{~h}$ using $\mathrm{Hg}$ lamp. Flash chromatography (02:98 EtOAc/Petroleum ether to 10:90 EtOAc/Petroleum ether) gave product $3 a$ as a white powder ( $87 \mathrm{mg}, 41 \%$ ).

Entry 3: General procedure B was applied to benzophenone I (160 mg, $0.88 \mathrm{mmol}$ ) and acrylonitrile 2 (1.33 mL, $20.24 \mathrm{mmol}$ ) in cyclohexane $(5.4 \mathrm{~mL}$ ) for $18 \mathrm{~h}$ using $300 \mathrm{~nm}$ monochromatic lamps in Rayonet apparatus. Flash chromatography (03:97 EtOAc/Petroleum ether to 5:95 EtOAc/Petroleum ether) gave product 3a as a white powder (70 mg, 32\%).

Entry 4: General procedure B was applied to benzophenone I (246 mg, $1.35 \mathrm{mmol}$ ) and acrylonitrile 2 (2.04 mL, $31.05 \mathrm{mmol}$ ) in cyclohexane $(8.2 \mathrm{~mL}$ ) for $18 \mathrm{~h}$ using $350 \mathrm{~nm}$ monochromatic lamps in Rayonet apparatus. Flash chromatography (03:97 EtOAc/Petroleum ether to 5:95 EtOAc/Petroleum ether) gave product 3a as a white powder (106 mg, 33\%).

Entry 5: General procedure A was applied to benzophenone I (228 mg, $1.25 \mathrm{mmol}$ ) and acrylonitrile 2 (1.74 mL, $26.4 \mathrm{mmol})$ in cyclohexane $(190 \mathrm{~mL})$ for $1.5 \mathrm{~h}$ using $\mathrm{Hg}$ lamp. Flash chromatography (03:97 EtOAc/Petroleum ether to 10:90 EtOAc/Petroleum ether) gave products $3 a(37 \mathrm{mg}, 13 \%)$ and 4 a (70 mg, 29\%) as a white powders. Entry 6: General procedure A was applied to benzophenone I (206 mg, $1.13 \mathrm{mmol}$ ) and acrylonitrile 2 (1.71 mL, $26.0 \mathrm{mmol})$ in cyclohexane $(200 \mathrm{~mL}$ ) for $3 \mathrm{~h}$ using $\mathrm{Hg}$ lamp. Flash chromatography (03:97 EtOAc/Petroleum ether to 5:95 EtOAc/Petroleum ether) gave product $4 \mathrm{a}$ as a white powder (75 $\mathrm{mg}, 32 \%)$.

Entry 7: General procedure A was applied to benzophenone I (911 mg, $5.0 \mathrm{mmol}$ ) and acrylonitrile 2 (7.57 mL, $115.0 \mathrm{mmol})$ in cyclohexane $(200 \mathrm{~mL})$ for $3 \mathrm{~h}$ using $\mathrm{Hg}$ lamp. Flash chromatography (01:99 EtOAc/Petroleum ether to 10:90 EtOAc/Petroleum ether) gave product 4a as a white powder (650 mg, 62\%).

Entry 8: General procedure A was applied to benzophenone I (1.775 mg, $9.75 \mathrm{mmol})$ and acrylonitrile 2 (14.73 $\mathrm{mL}, 223.8 \mathrm{mmol})$ in cyclohexane $(200 \mathrm{~mL})$ for $3 \mathrm{~h}$ using $\mathrm{Hg}$ lamp. Flash chromatography (05:95 EtOAc/Petroleum ether to 10:90 EtOAc/Petroleum ether) gave products $3 a(160 \mathrm{mg}, 7 \%)$ and $4 \mathrm{a}$ ( $871 \mathrm{mg}, 43 \%)$ as a white powders. Entry 9: General procedure A was applied to benzophenone I (911 mg, $5.0 \mathrm{mmol}$ ) and acrylonitrile 2 (2.28 mL, $35.0 \mathrm{mmol})$ in cyclohexane $(200 \mathrm{~mL})$ for $3 \mathrm{~h}$ using $\mathrm{Hg}$ lamp. Flash chromatography (5:95 EtOAc/Petroleum ether) gave product $4 \mathrm{a}$ as a white powder (292 $\mathrm{mg}, 28 \%)$.

Entry 10: General procedure A was applied to benzophenone I (911 mg, $5.0 \mathrm{mmol})$ and acrylonitrile 2 (15.0 mL, $230.0 \mathrm{mmol})$ in cyclohexane $(200 \mathrm{~mL})$ for $3 \mathrm{~h}$ using $\mathrm{Hg}$ lamp. Flash chromatography (5:95 EtOAc/Petroleum ether) gave products $3 a(51 \mathrm{mg}, 4 \%)$ and $\mathbf{4 a}(301 \mathrm{mg}, 29 \%)$ as a white powders.

Entry 11: General procedure A was applied to benzophenone I (111 mg, $0.61 \mathrm{mmol})$ and acrylonitrile 2 (976 $\mu \mathrm{L}$, $14.8 \mathrm{mmol}$ ) in cyclohexane $(25 \mathrm{~mL})$ for $3 \mathrm{~h}$ using $350 \mathrm{~nm}$ monochromatic lamps in Rayonet apparatus. Flash 
chromatography (04:96 EtOAc/Petroleum ether to 8:92 EtOAc/Petroleum ether) gave products $3 a$ ( $27 \mathrm{mg}, 18 \%)$ and $4 \mathrm{a}$ ( $28 \mathrm{mg}, 22 \%)$ as a white powders.

Entry 12: General procedure A was applied to benzophenone I (784 mg, $4.3 \mathrm{mmol}$ ) and acrylonitrile 2 (6.51 mL, $98.9 \mathrm{mmol}$ ) in benzene $(180 \mathrm{~mL})$ for $3 \mathrm{~h}$ using $\mathrm{Hg}$ lamp. Flash chromatography (05:95 EtOAc/Petroleum ether to 10:90 EtOAc/Petroleum ether) gave product 4a as a white powder (392 mg, 44\%).

Entry 13: General procedure A was applied to benzophenone I (911 mg, $5.0 \mathrm{mmol})$ and acrylonitrile $2(7.57 \mathrm{~mL}$, $115.0 \mathrm{mmol})$ in toluene $(200 \mathrm{~mL})$ for $3 \mathrm{~h}$ using $\mathrm{Hg}$ lamp. Flash chromatography (03:97 EtOAc/Petroleum ether to 10:90 EtOAc/Petroleum ether) gave product 4a as a white powder (324 mg, 31\%).

In all cases, Spectral data were identical to those described above.

Domino photochemical reaction in tetrahydrofuran (Table 1, entry 14)

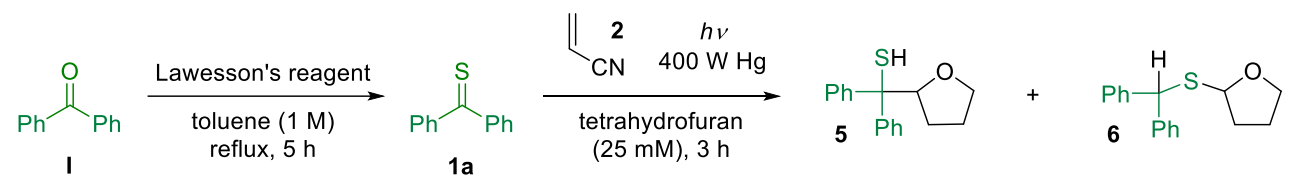

General procedure A was applied to benzophenone I (784 mg, $4.3 \mathrm{mmol}$ ) and acrylonitrile 2 (6.51 mL, $98.9 \mathrm{mmol})$ in tetrahydrofuran (180 mL) for $3 \mathrm{~h}$ using $\mathrm{Hg}$ lamp. Flash chromatography (02:98 EtOAc/Petroleum ether to 05:95 EtOAc/Petroleum ether) gave the two products 5 (272 mg, 23\%) and 6 (228 mg, 20\%) as colorless oils.

\section{Diphenyl(tetrahydrofuran-2-yl)methanethiol 5}
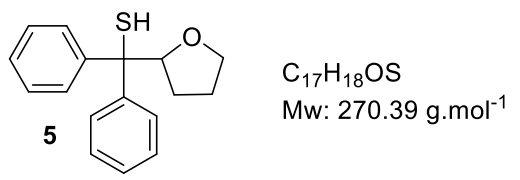

$\mathbf{R}_{\mathbf{f}}$ (06:94, EtOAc/Petroleum Ether): 0.42. IR v (cm $\left.{ }^{-1}\right): 3022,3054,2963,2859$, 2557, 1657, 1598, 1491, 1442, 1277, 1067, 927, 753, 694. ${ }^{1} \mathbf{H}$ NMR (360 MHz, $\left.\mathrm{CDCl}_{3}\right): \delta$ 7.57-7.14 (m, $\left.10 \mathrm{H}\right), 4.89(\mathrm{dd}, J=8.0,6.4 \mathrm{~Hz}, 1 \mathrm{H}), 3.96-3.77(\mathrm{~m}, 2 \mathrm{H})$,

$2.47(\mathrm{~s}, 1 \mathrm{H}), 1.93-1.55(\mathrm{~m}, 4 \mathrm{H}) .{ }^{13} \mathrm{C}$ NMR $\left(90 \mathrm{MHz}, \mathrm{CDCl}_{3}\right): \delta 145.2(2 \mathrm{C}), 129.1(2 \mathrm{CH}), 128.1(2 \mathrm{CH}), 128.0(2 \mathrm{CH})$, $127.8(2 \mathrm{CH}), 126.9(\mathrm{CH}), 126.6(\mathrm{CH}), 84.0(\mathrm{CH}), 69.9\left(\mathrm{CH}_{2}\right), 62.7(\mathrm{C}), 29.2\left(\mathrm{CH}_{2}\right), 26.1\left(\mathrm{CH}_{2}\right)$. HRMS (ESI-TOF) $\mathrm{m} / z$ : $[\mathrm{M}+\mathrm{Na}]^{+} \mathrm{Calcd}$ for $\mathrm{C}_{17} \mathrm{H}_{18} \mathrm{OSNa}$ 293.0976. Found 293.0982. Spectral data were identical to those described in the literature. $^{\mathrm{s} 2}$

\section{2-(Benzhydrylthio)tetrahydrofuran 6}

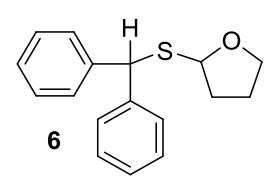

$\mathrm{C}_{17} \mathrm{H}_{18} \mathrm{OS}$ Mw: $270.39 \mathrm{~g} \cdot \mathrm{mol}^{-1}$

$\mathbf{R}_{\mathbf{f}}$ (06:94, EtOAc/Petroleum Ether): 0.33. IR $\mathbf{v}\left(\mathrm{cm}^{-1}\right):$ 3059, 3025, 2975, 2947, 2871, 1658, 1598, 1491, 1447, 1278, 1046, 905, 745, 694. ${ }^{1}$ H NMR

(360 MHz, $\left.\mathrm{CDCl}_{3}\right): \delta$ 7.55-7.13 (m, $\left.10 \mathrm{H}\right), 5.38(\mathrm{~s}, 1 \mathrm{H}), 5.04(\mathrm{dd}, J=7.3,3.5$

$\mathrm{Hz}, 1 \mathrm{H}), 4.04-3.86(\mathrm{~m}, 2 \mathrm{H}), 2.20-1.70(\mathrm{~m}, 4 \mathrm{H}) .{ }^{13} \mathrm{C}$ NMR (90 MHz, $\left.\mathrm{CDCl}_{3}\right): \delta 141.8$ (C), 141.7 (C), $128.7(2 \mathrm{CH}), 128.6$

\footnotetext{
S2 Kito, N.; Ohno, A. Photochemical reaction of diaryl thioketones in ethereal solutions. A stable transient free-radical. Bull. Chem. Soc. Jpn. 1973, 46, 2487-2489.
} 
(2CH), $128.5(2 \mathrm{CH}), 128.2(2 \mathrm{CH}), 127.2(\mathrm{CH}), 127.0(\mathrm{CH}), 83.4(\mathrm{CH}), 66.9\left(\mathrm{CH}_{2}\right), 52.6(\mathrm{CH}), 32.0\left(\mathrm{CH}_{2}\right), 24.8\left(\mathrm{CH}_{2}\right)$. HRMS (ESI-TOF) $\mathrm{m} / \mathrm{z}$ : [M + Na] $]^{+}$Calcd for $\mathrm{C}_{17} \mathrm{H}_{18} \mathrm{OSNa}$ 293.0976. Found 293.0963. Spectral data were identical to those described in the literature. ${ }^{\mathrm{S2}}$

\section{Photochemical ring enlargement reaction in different experimental conditions (Table 2, entries 1-6)}

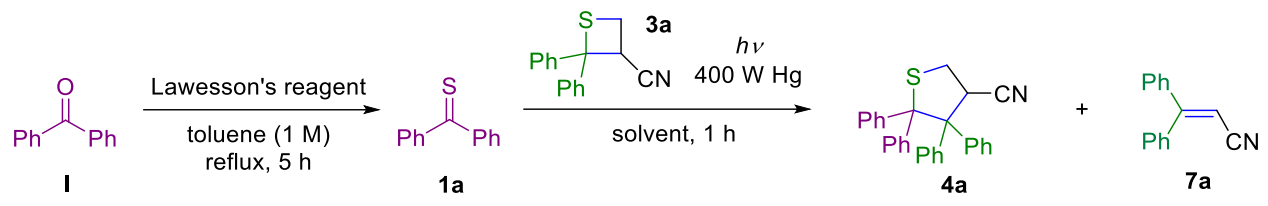

Entry 1: General procedure C was applied to benzophenone I (53 mg, $0.21 \mathrm{mmol}$ ) without thietane $3 a$ in cyclohexane $(200 \mathrm{~mL})$ for $3 \mathrm{~h}$ using $\mathrm{Hg}$ lamp. Flash chromatography (02:98 EtOAc/Petroleum ether to 10:90 EtOAc/Petroleum ether) gave alkene $7 \mathrm{a}(37 \mathrm{mg}, 85 \%)$.

Entry 2: General procedure $\mathbf{C}$ was applied to benzophenone I (34 mg, $0.19 \mathrm{mmol}, 1$ equiv.) and thietane $3 a$ (47 $\mathrm{mg}, 0.19 \mathrm{mmol})$ in cyclohexane $(200 \mathrm{~mL})$ for $3 \mathrm{~h}$ using $\mathrm{Hg}$ lamp. Flash chromatography (04:96 EtOAc/Petroleum ether to 06:94 EtOAc/Petroleum ether) gave product 4a as a white powder (43 mg, 55\%) and alkene 7a (12 mg, $31 \%)$.

Entry 3: General procedure C was applied to benzophenone I (58 mg, $0.32 \mathrm{mmol} 2$ equiv.) and thietane $3 a$ (39 $\mathrm{mg}, 0.16 \mathrm{mmol})$ in cyclohexane $(200 \mathrm{~mL})$ for $1 \mathrm{~h}$ using $\mathrm{Hg}$ lamp. Flash chromatography (04:96 EtOAc/Petroleum ether to 06:94 EtOAc/Petroleum ether) gave product 4a as white powder (50 mg, 76\%).

Entry 4: General procedure $\mathbf{C}$ was applied to benzophenone I (123 mg, $0.68 \mathrm{mmol}, 2$ equiv.) and thietane $3 a$ (85 $\mathrm{mg}, 0.34 \mathrm{mmol}, 130 \mathrm{mM}$ ) in cyclohexane $(2.6 \mathrm{~mL})$ for $4 \mathrm{~h}$ using $\mathrm{Hg}$ lamp. Flash chromatography (02:98 EtOAc/Petroleum ether to 08:92 EtOAc/Petroleum ether) gave alkene 7a (53 mg, 76\%).

Entry 5: General procedure $\mathbf{C}$ was applied to benzophenone I (58 mg, $0.32 \mathrm{mmol}, 2$ equiv.) and thietane $3 a$ (39 $\mathrm{mg}, 0.16 \mathrm{mmol})$ in cyclohexane $(200 \mathrm{~mL})$ was warmed up in the dark from room temperature to $80{ }^{\circ} \mathrm{C}$ over $5 \mathrm{~h}$ using an oil bath. Thiobenzophenone 1a and thietane 3a were recovered entirely.

Entry 6: General procedure $\mathbf{C}$ was applied to benzophenone I (58 mg, $0.32 \mathrm{mmol}, 2$ equiv.) and thietane $3 a$ (39 $\mathrm{mg}, 0.16 \mathrm{mmol})$ in cyclohexane $(200 \mathrm{~mL})$ was warmed up in the dark from room temperature to $80^{\circ} \mathrm{C}$ over $5 \mathrm{~h}$ in the presence of AIBN (79 mg, $0.48 \mathrm{mmol}, 3$ equiv.) using an oil bath. Thiobenzophenone 1a and thietane 3a were recovered entirely.

In all cases, spectral data were identical to those described above. 


\section{Rhodium-catalyzed thia-Stevens rearrangement of thietane 3a (Scheme 5)}

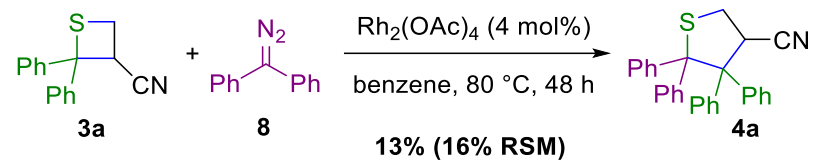

Following the literature procedure, ${ }^{\mathrm{S3}}$ diazo compound 8 (46 mg, $0.24 \mathrm{mmol}, 1.1$ equiv.) and $\mathrm{Rh}_{2}(\mathrm{OAc})_{4}(4 \mathrm{mg}$, $0.009 \mathrm{mmol}, 4 \mathrm{~mol} \%)$ were added to a solution of thietane $3 a(54 \mathrm{mg}, 0.22 \mathrm{mmol})$ in benzene $(2.5 \mathrm{~mL})$ at room temperature under argon. The reaction mixture was stirred at room temperature for $5 \mathrm{~min}$ and then at reflux for $48 \mathrm{~h}$ under argon using an oil bath. The solution was then evaporated reduced pressure to give the crude product, which was purified by flash chromatography (03:97 EtOAc/Petroleum ether) to afford product $4 a$ as a white powder (12 mg, 13\%). In addition, unreacted 3a was recovered (11 mg, 20\%).

Spectral data were identical to those described above.

\footnotetext{
s3 Nair, V.; Nair, S. M.; Mathai, S.; Liebscher, J.; Ziemer, B.; Narsimulu, K. The Rh(II) catalyzed reaction of diethyl diazomalonate with thietanes: a facile synthesis of tetrahydrothiophene derivatives via sulfonium ylides. Tetrahedron Lett. 2004, 45, 5759-5762.
} 
3. ${ }^{1} \mathrm{H}$ and ${ }^{13} \mathrm{C}$ NMR spectra of "homo" tetra-aryl tetrahydrothiophenes 4a-e (Scheme 1):

\section{4,4,5,5-Tetraphenyltetrahydrothiophene-3-carbonitrile 4a}

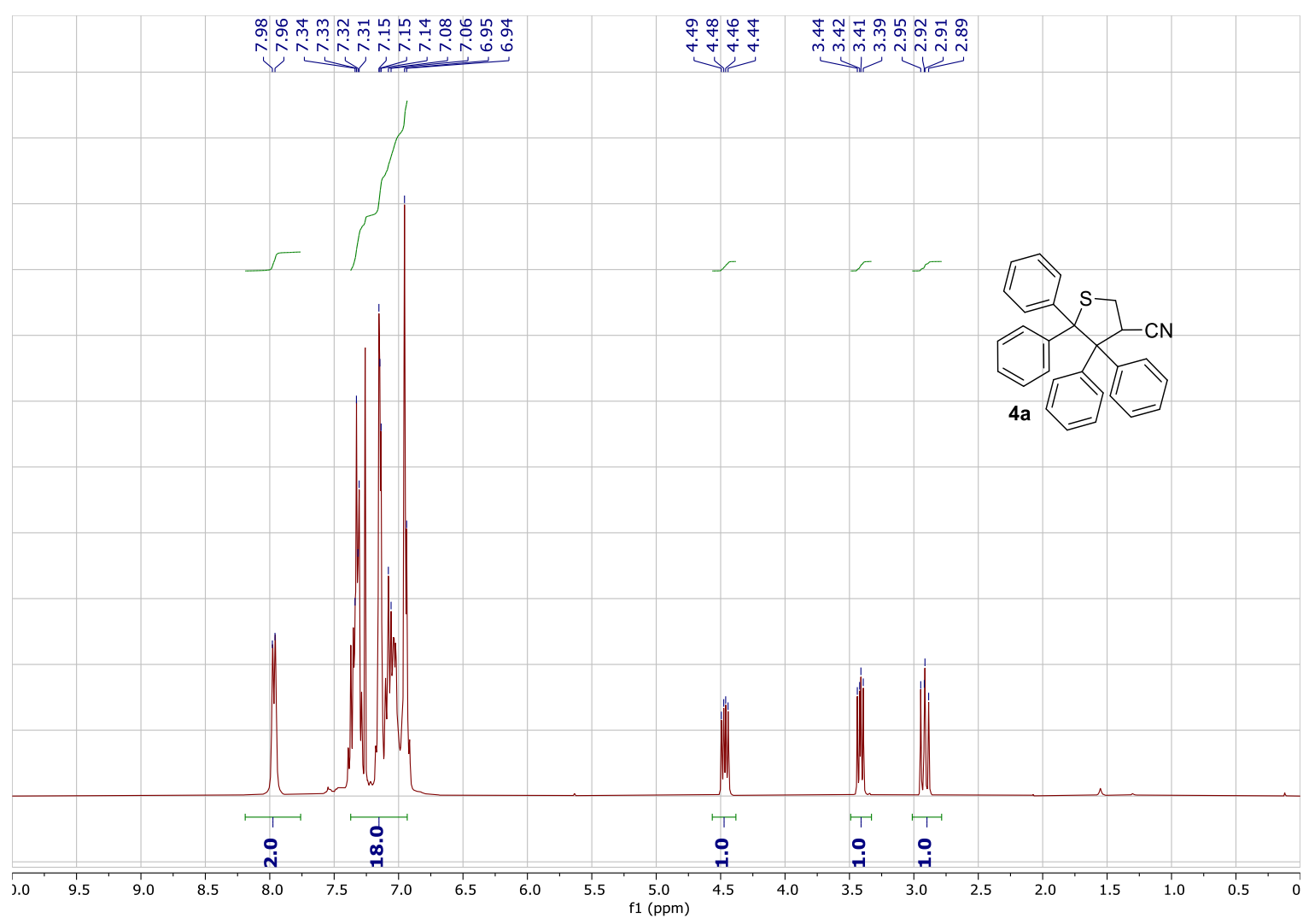

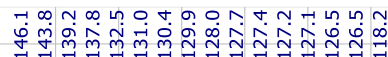

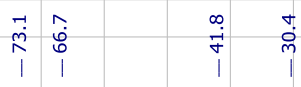

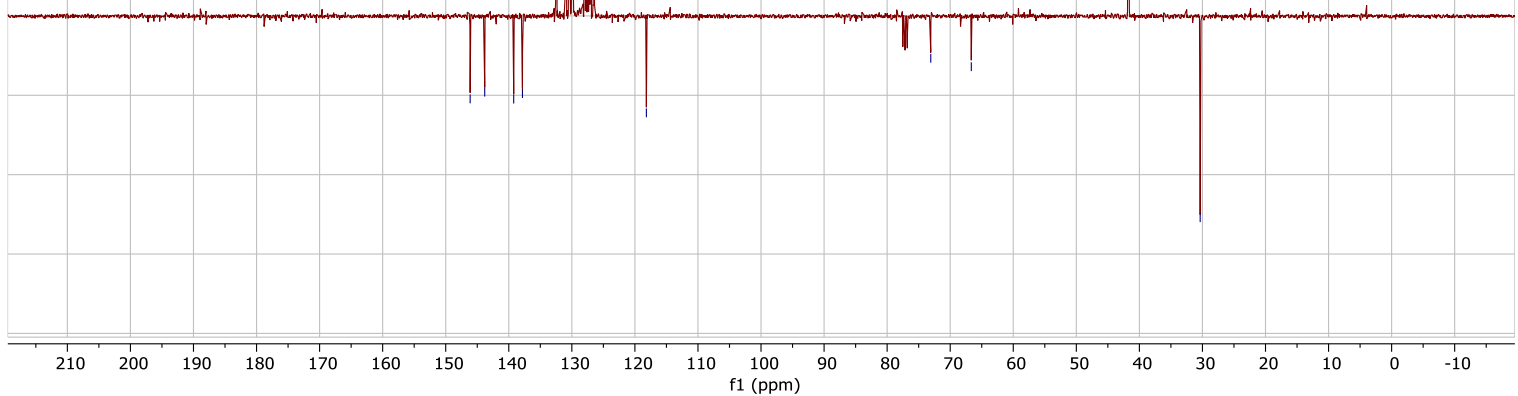


4,4,5,5-Tetra-p-tolyltetrahydrothiophene-3-carbonitrile 4b
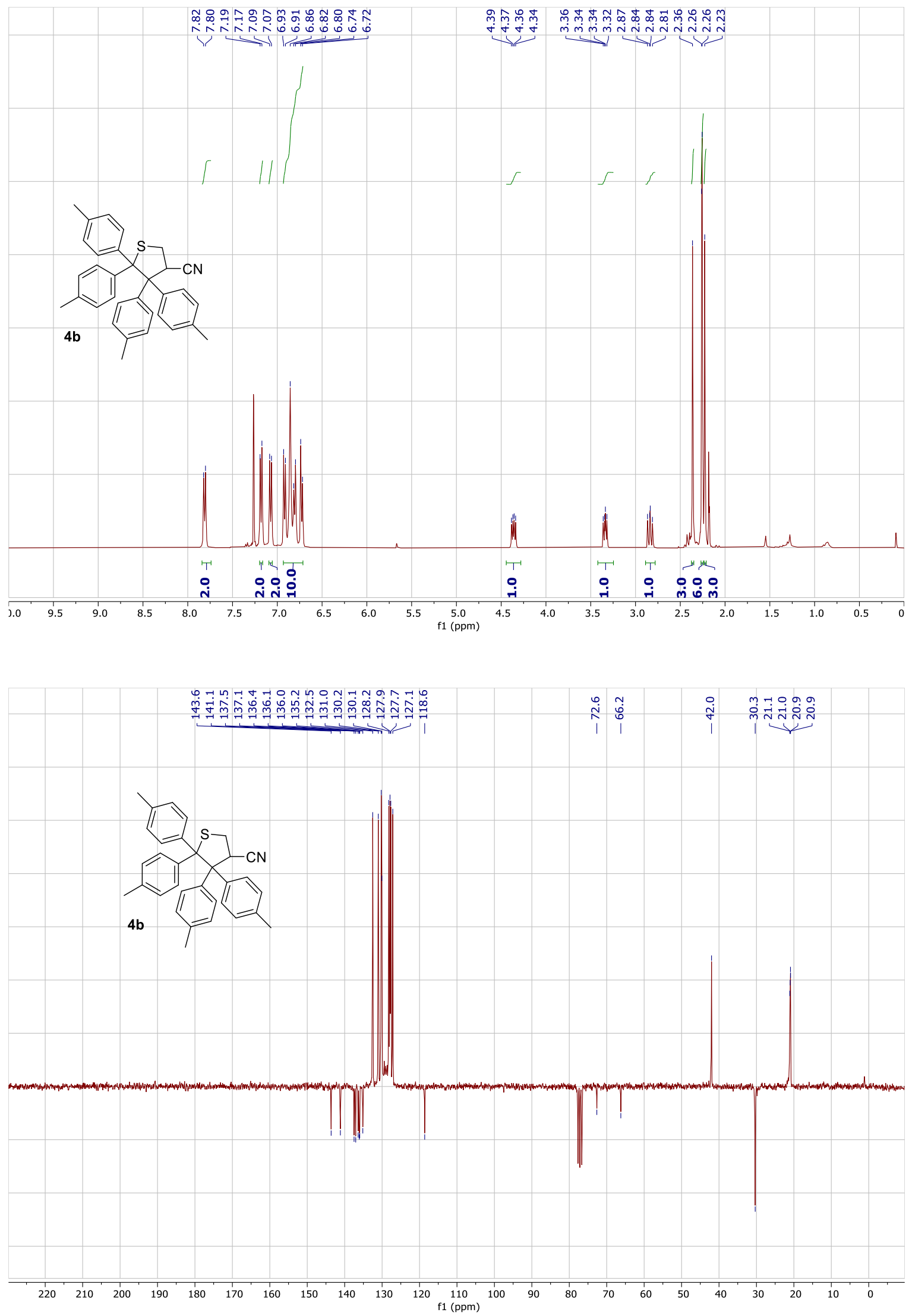
4,4,5,5-Tetrakis(4-chlorophenyl)tetrahydrothiophene-3-carbonitrile 4c
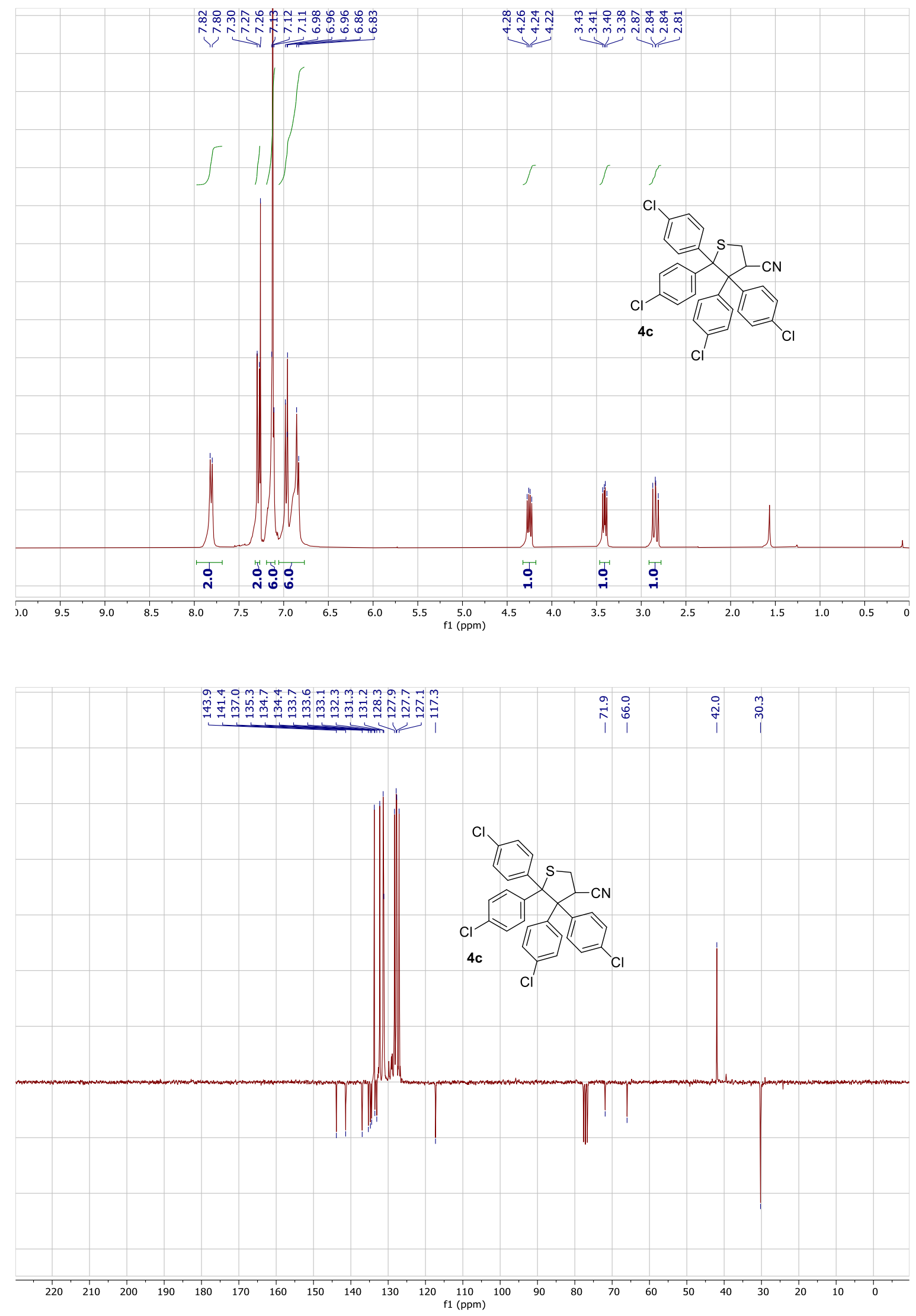
4,4,5,5-Tetrakis(4-methoxyphenyl)tetrahydrothiophene-3-carbonitrile 4d
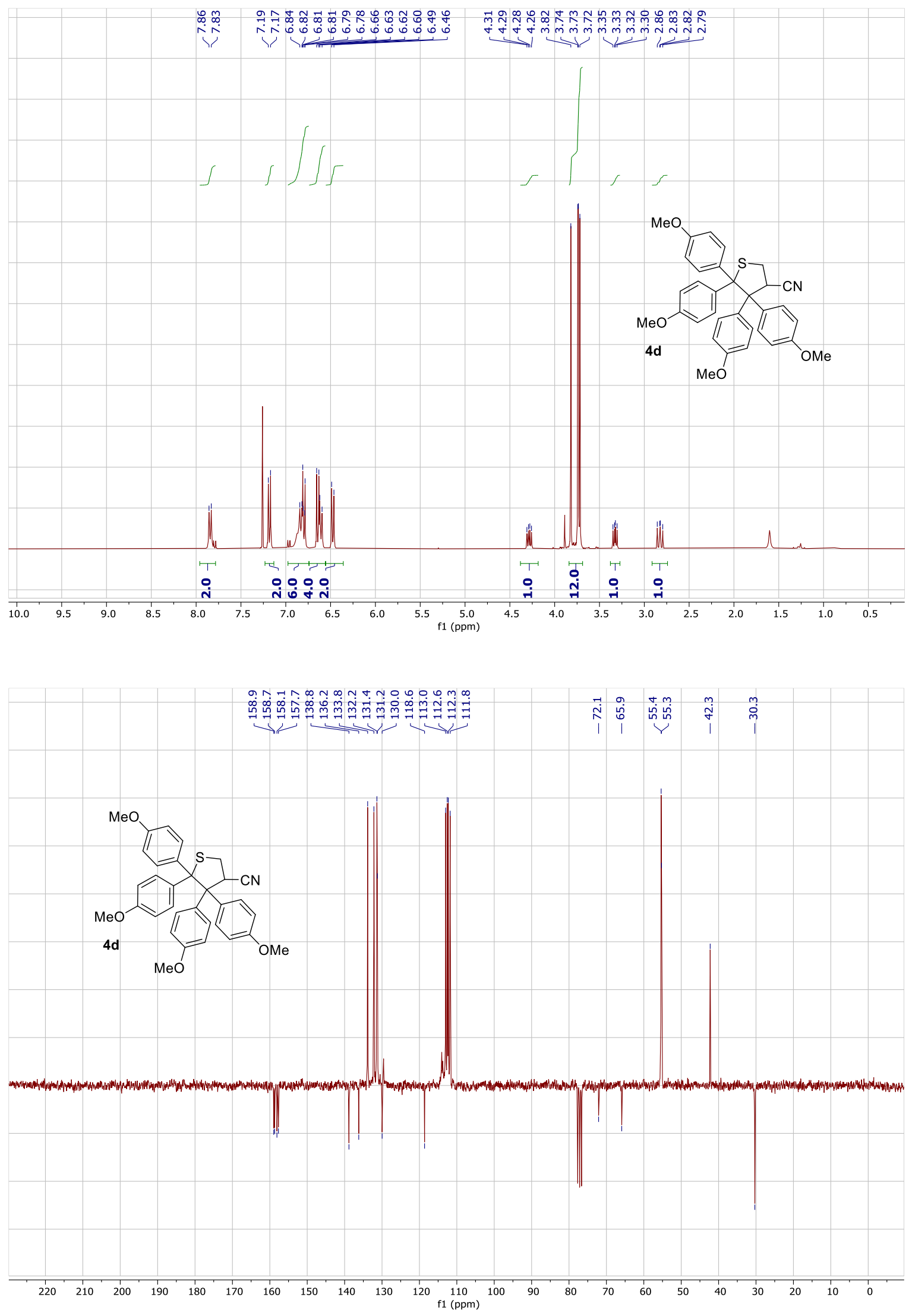
4,4,5,5-Tetrakis(4-fluorophenyl)tetrahydrothiophene-3-carbonitrile 4e

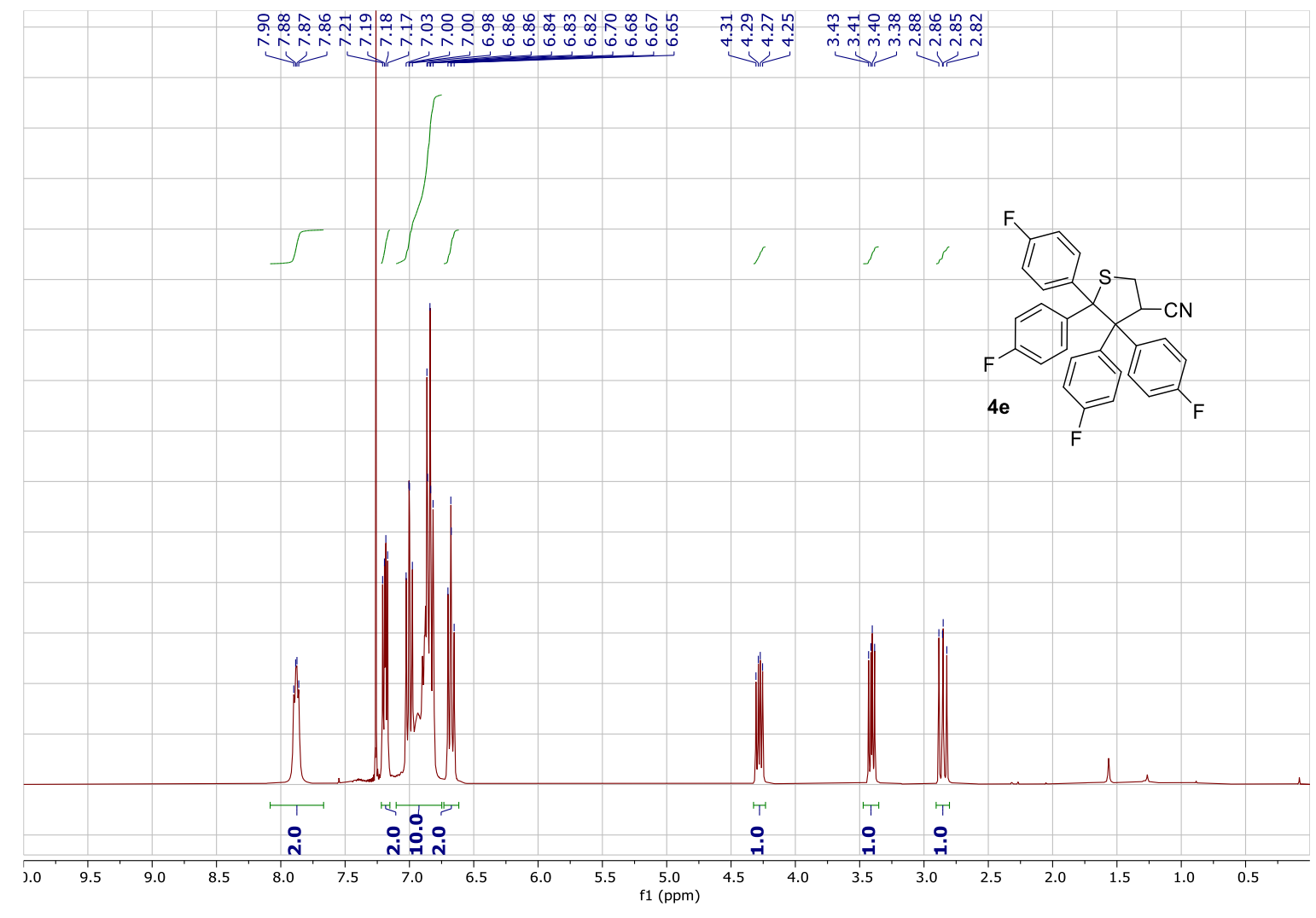

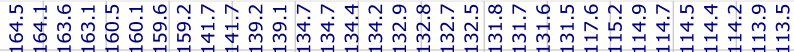

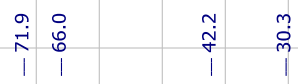

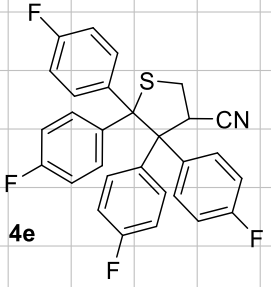

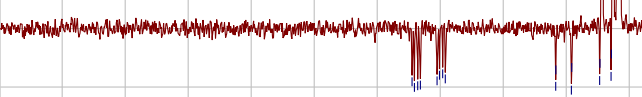
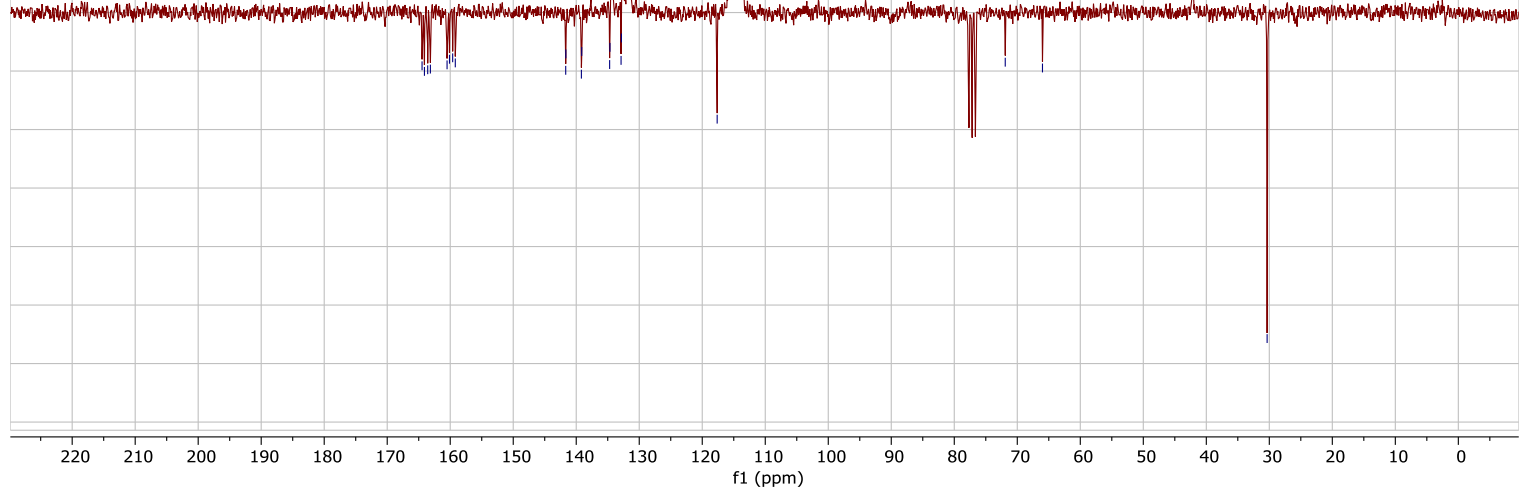
4. ${ }^{1} \mathrm{H}$ and ${ }^{13} \mathrm{C}$ NMR spectra of thietanes 3a-c (Scheme 2):

\section{2,2-Diphenylthietane-3-carbonitrile 3a}
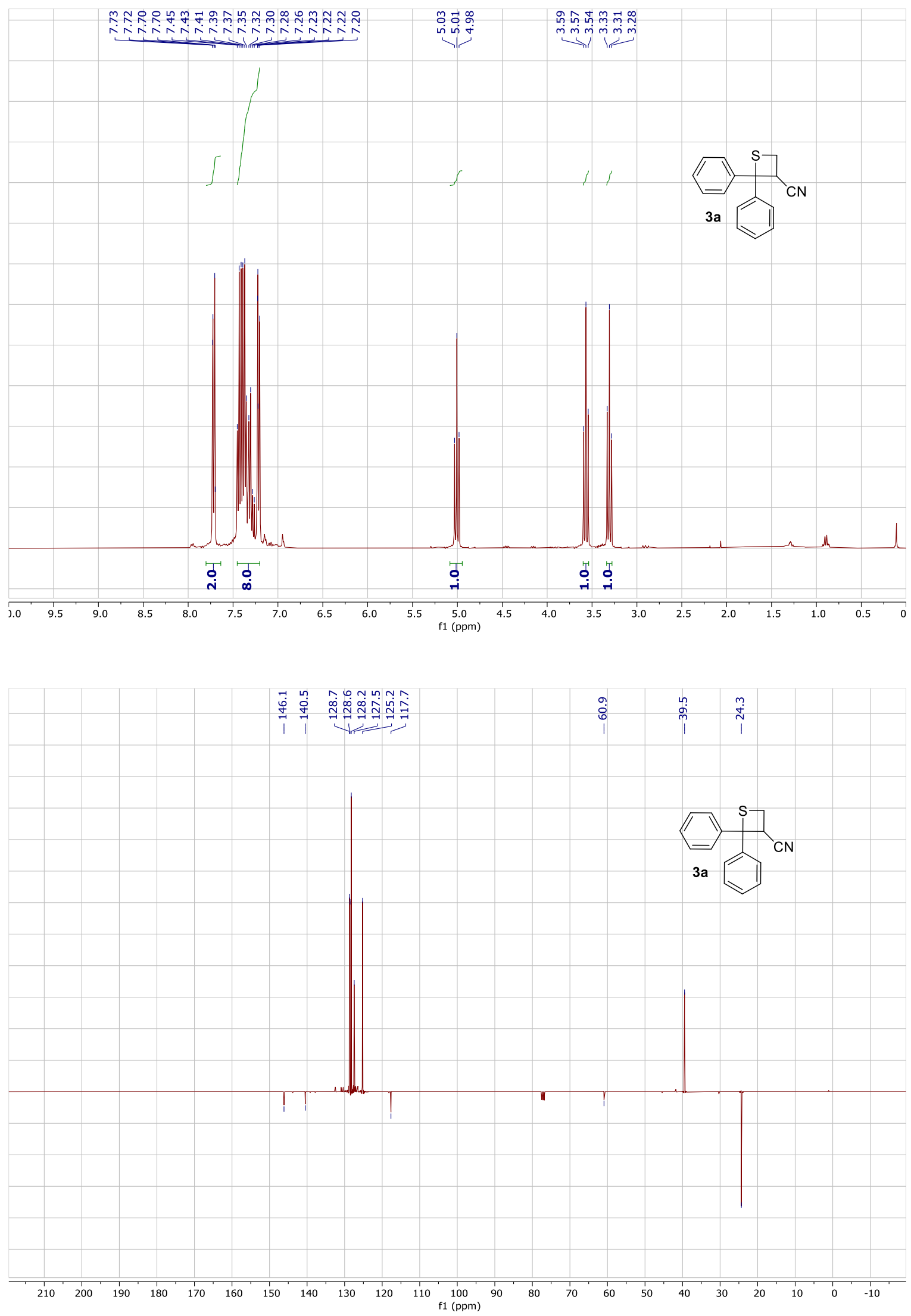
2,2-Di-p-tolylthietane-3-carbonitrile 3b
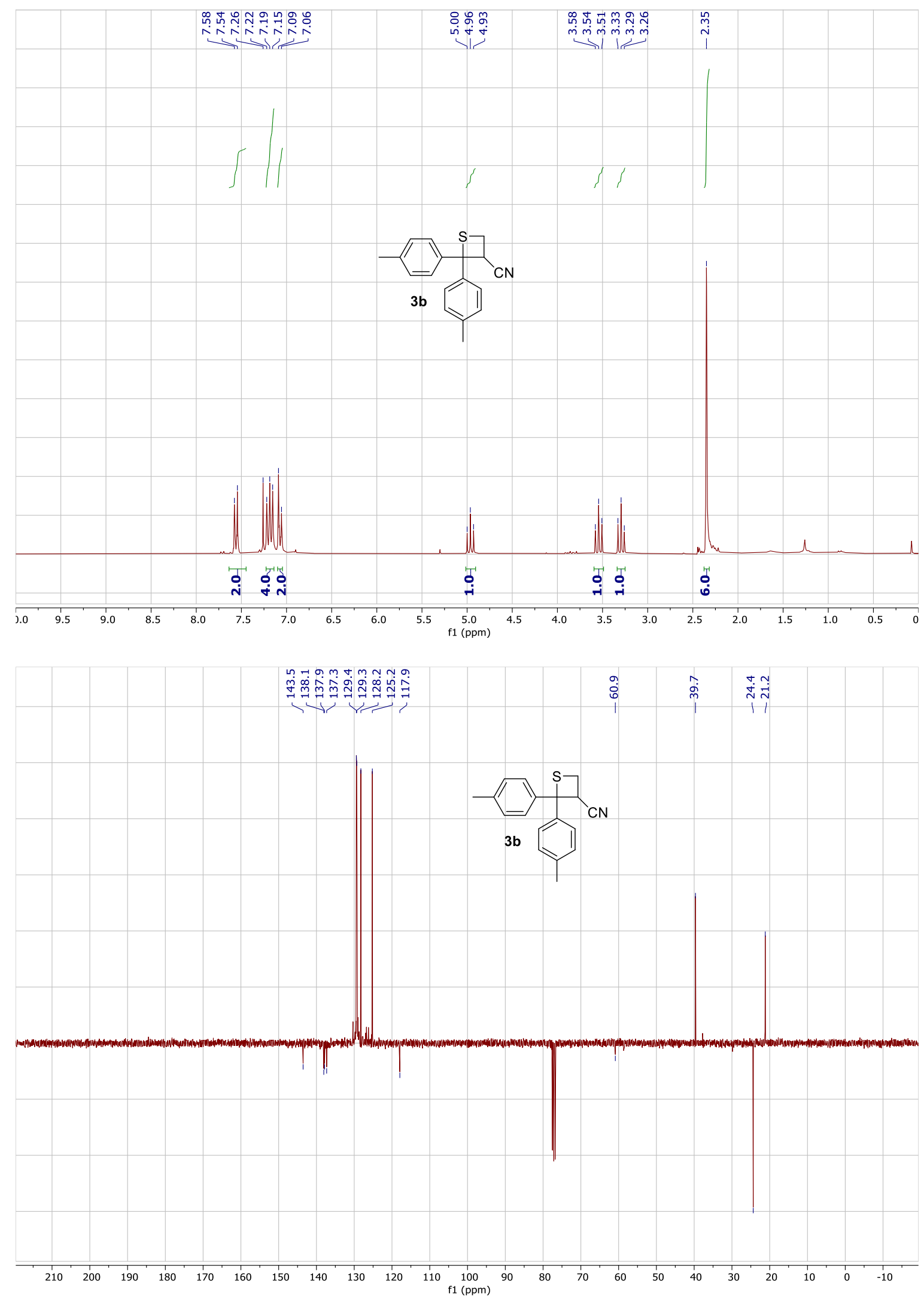
2,2-Bis(4-chlorophenyl)thietane-3-carbonitrile 3c
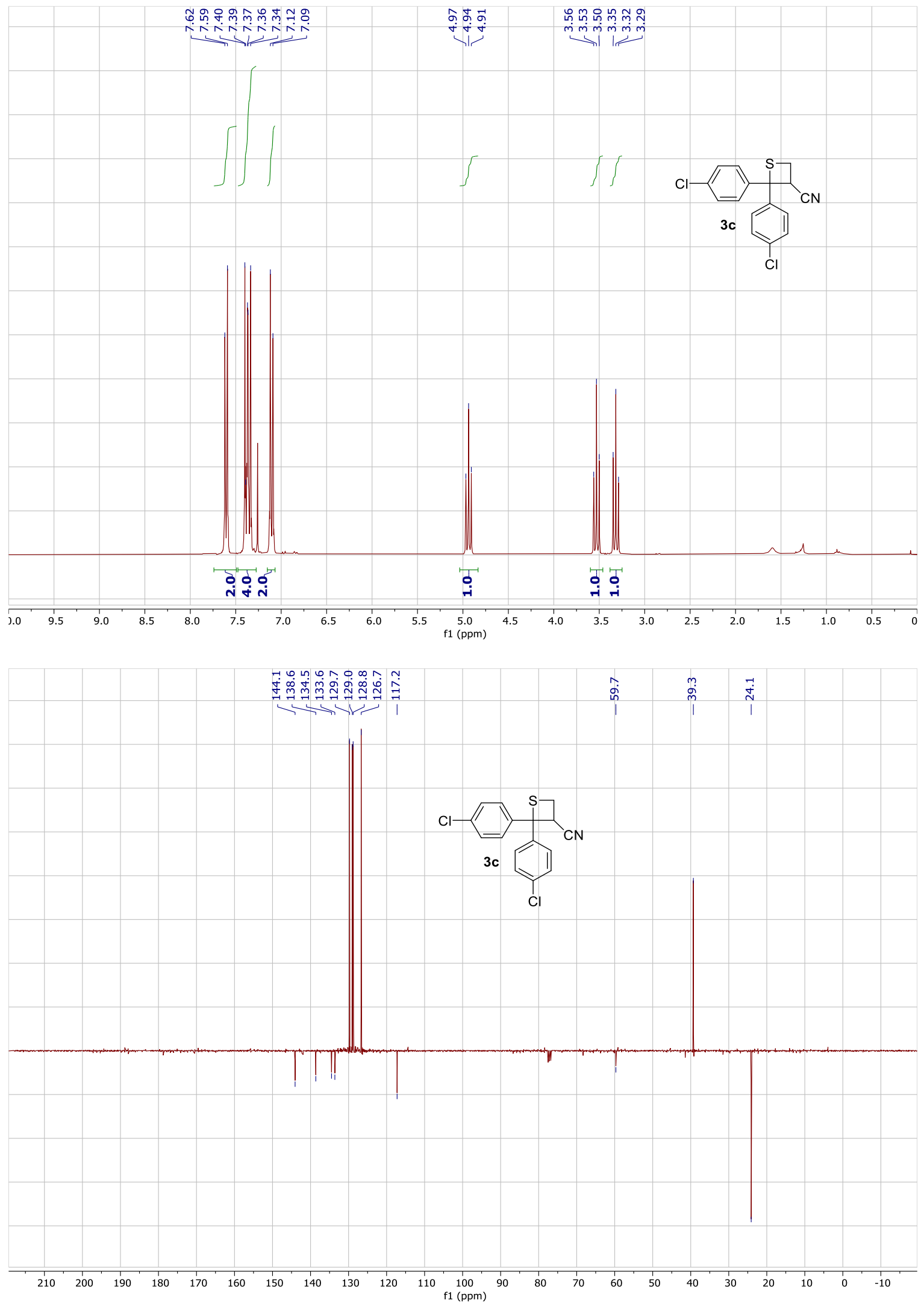
5. ${ }^{1} \mathrm{H}$ and ${ }^{13} \mathrm{C}$ NMR spectra of "hetero" tetra-aryl tetrahydrothiophenes 4 (Scheme 3):

\section{4,4-Diphenyl-5,5-di-p-tolyltetrahydrothiophene-3-carbonitrile 4ab}

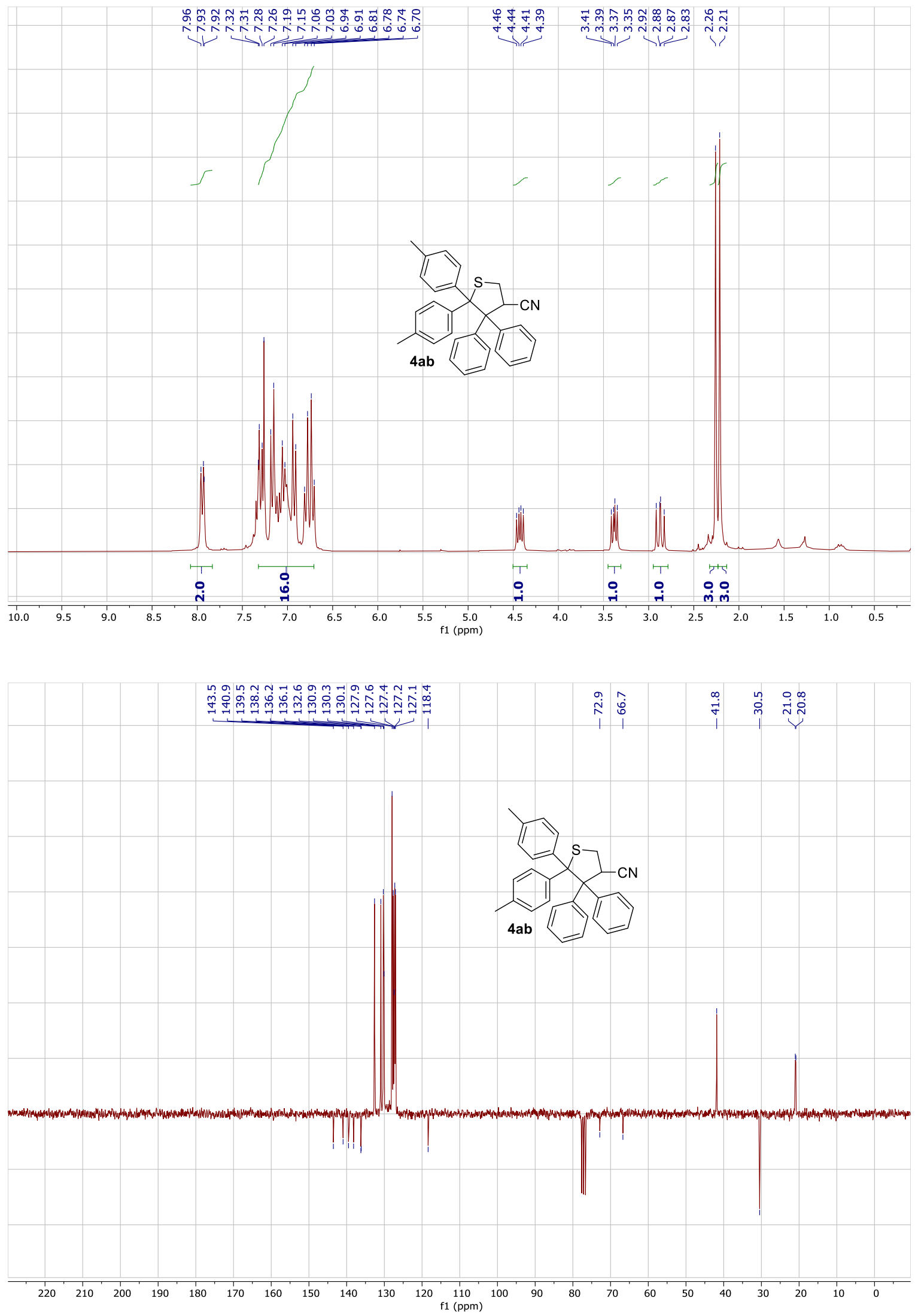




\section{5,5-Bis(4-chlorophenyl)-4,4-diphenyltetrahydrothiophene-3-carbonitrile 4ac}

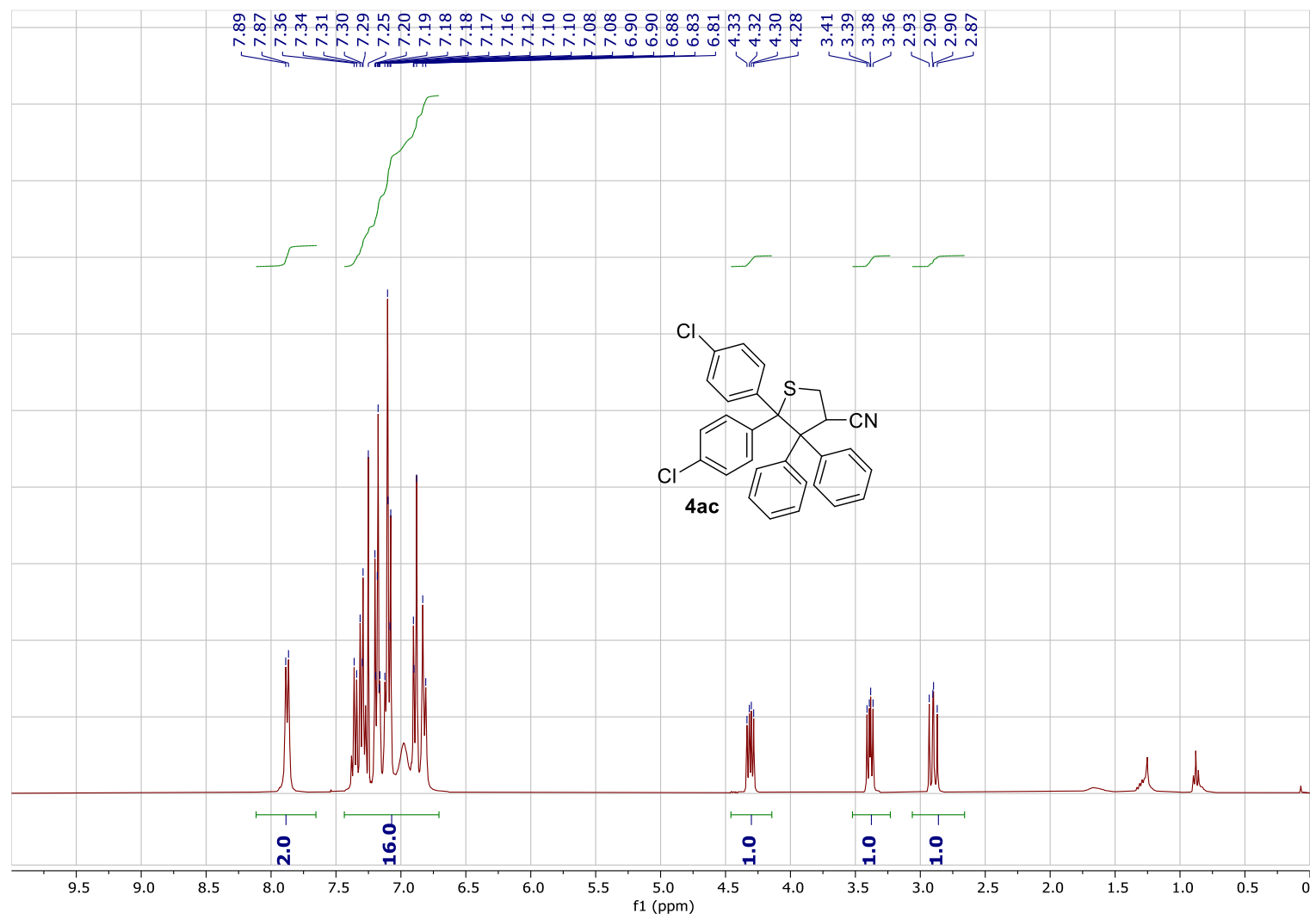

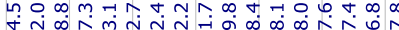
寸 ₹.

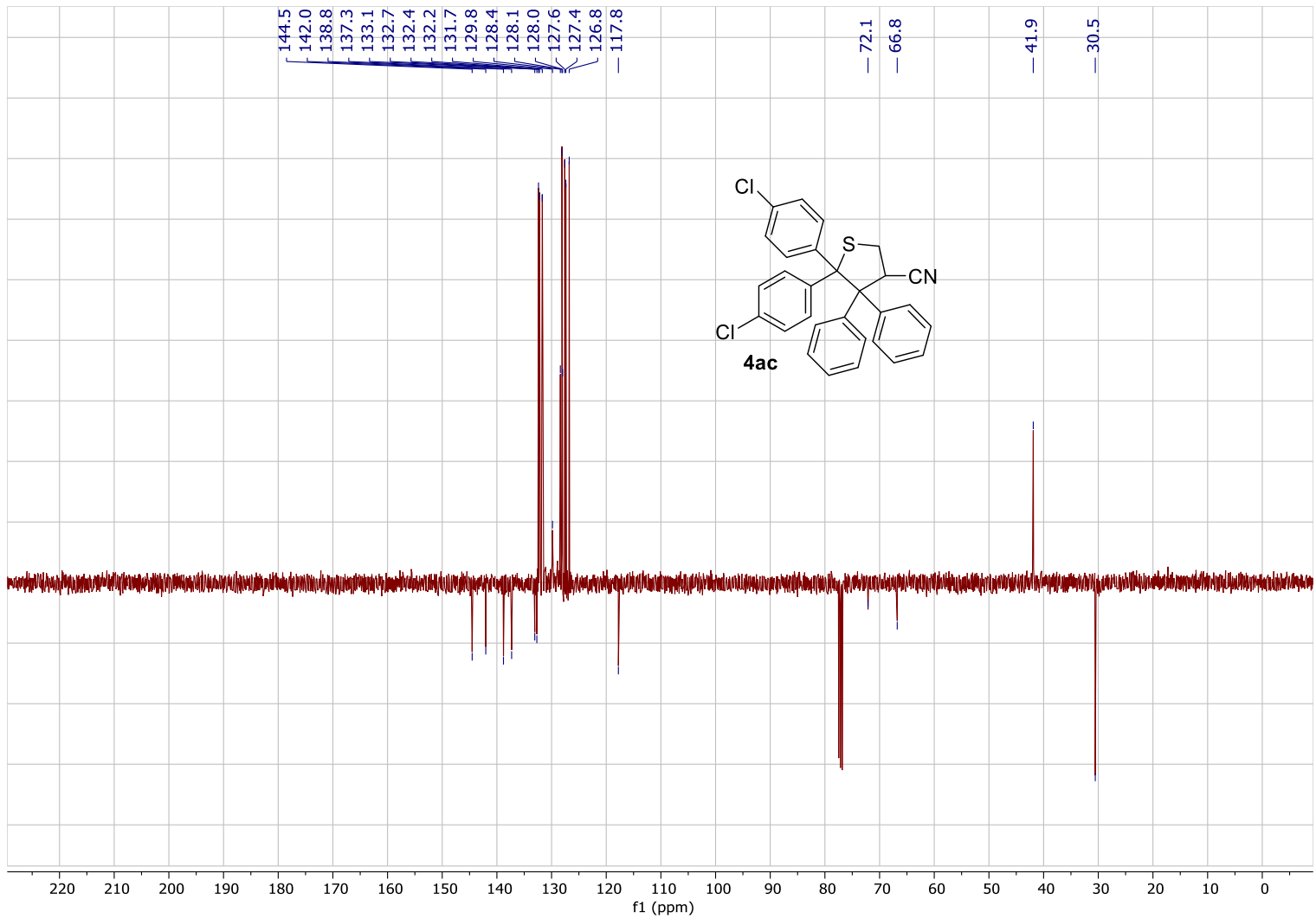




\section{5,5-Bis(4-methoxyphenyl)-4,4-diphenyltetrahydrothiophene-3-carbonitrile 4ad}
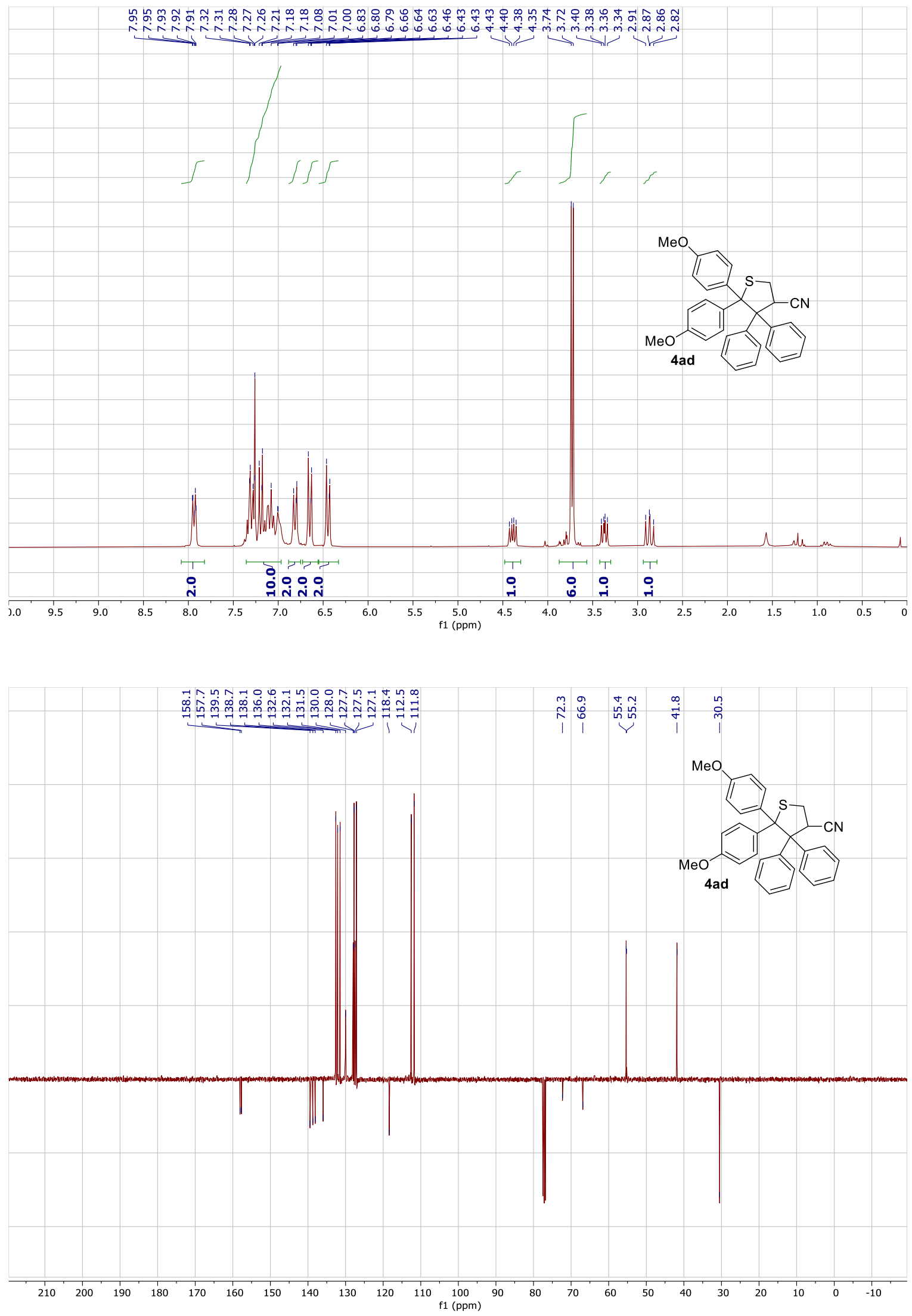


\section{5,5-Bis(4-fluorophenyl)-4,4-diphenyltetrahydrothiophene-3-carbonitrile 4ae}
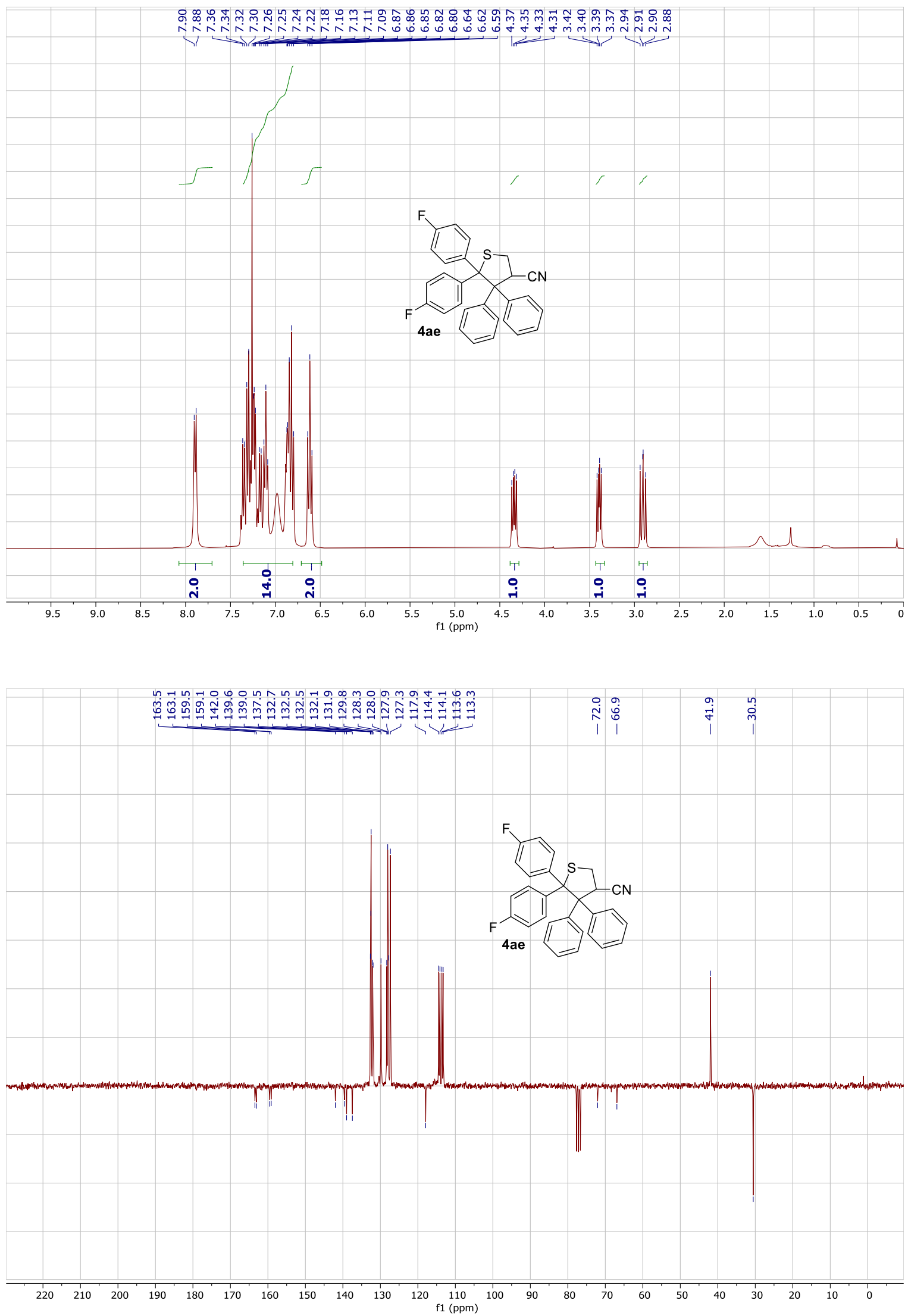
5,5-Diphenyl-4,4-di-p-tolyltetrahydrothiophene-3-carbonitrile 4ba
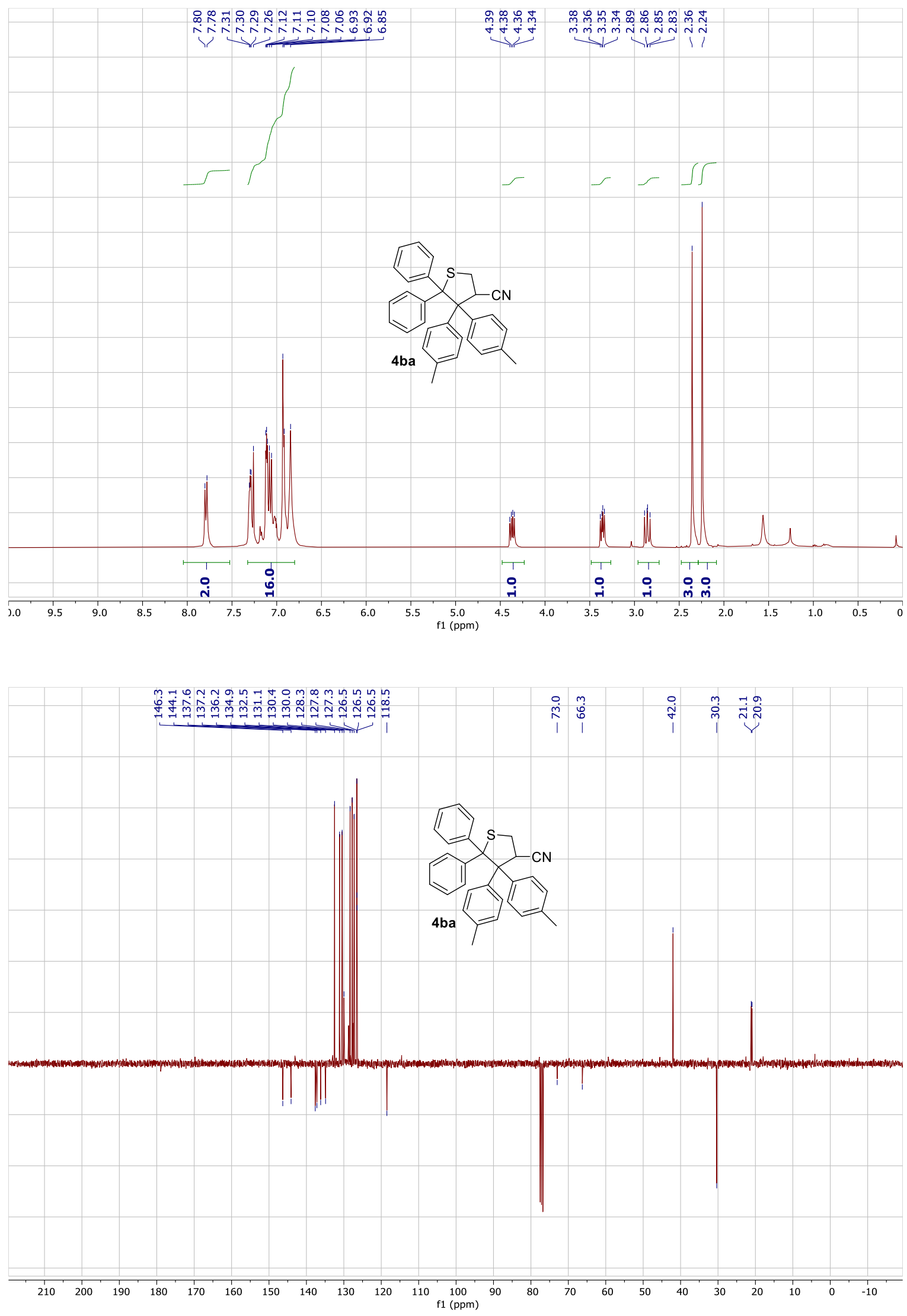


\section{4,4-Bis(4-chlorophenyl)-5,5-diphenyltetrahydrothiophene-3-carbonitrile 4ca}
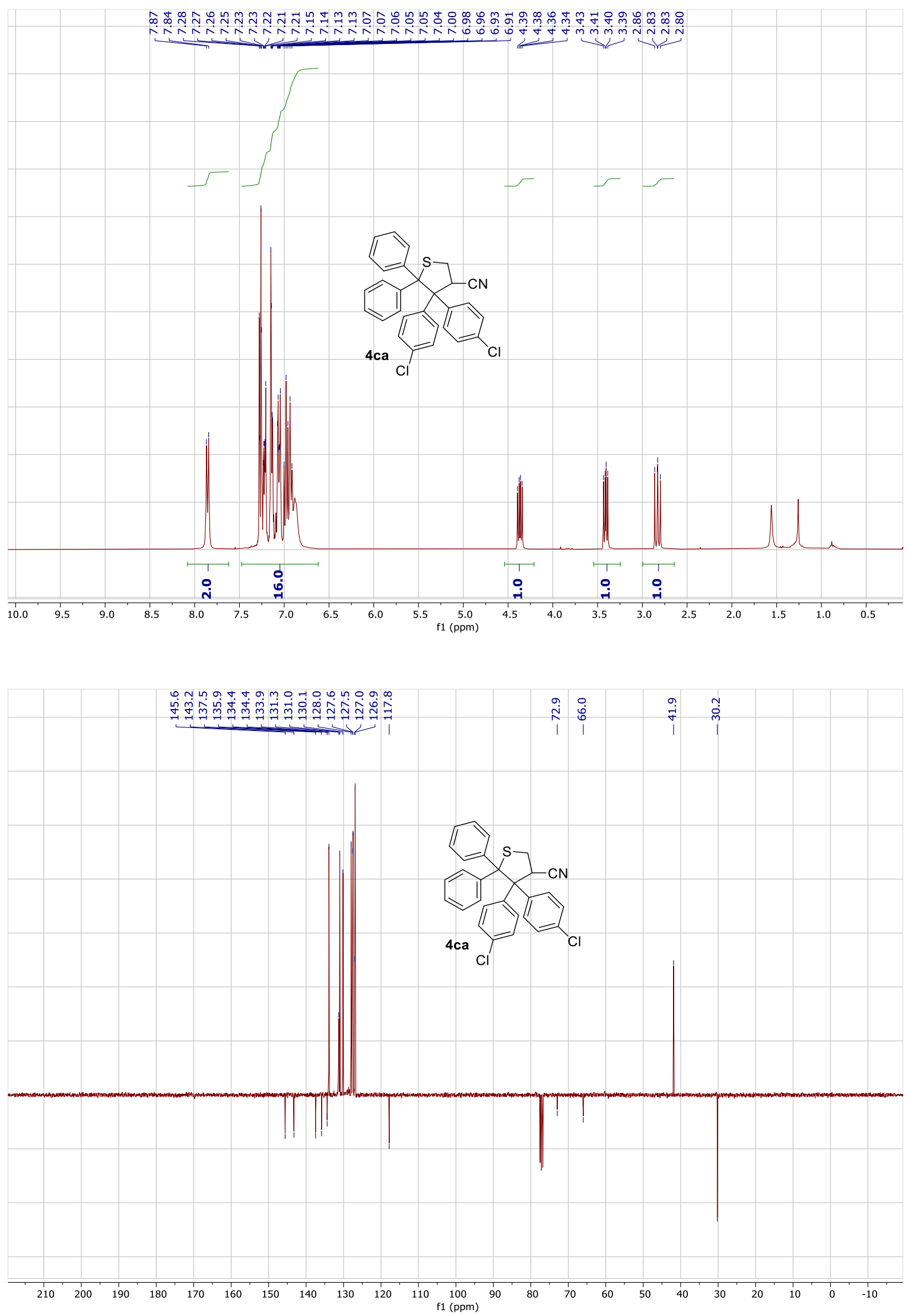
4,4-Bis(4-chlorophenyl)-5,5-di-p-tolyltetrahydrothiophene-3-carbonitrile 4cb
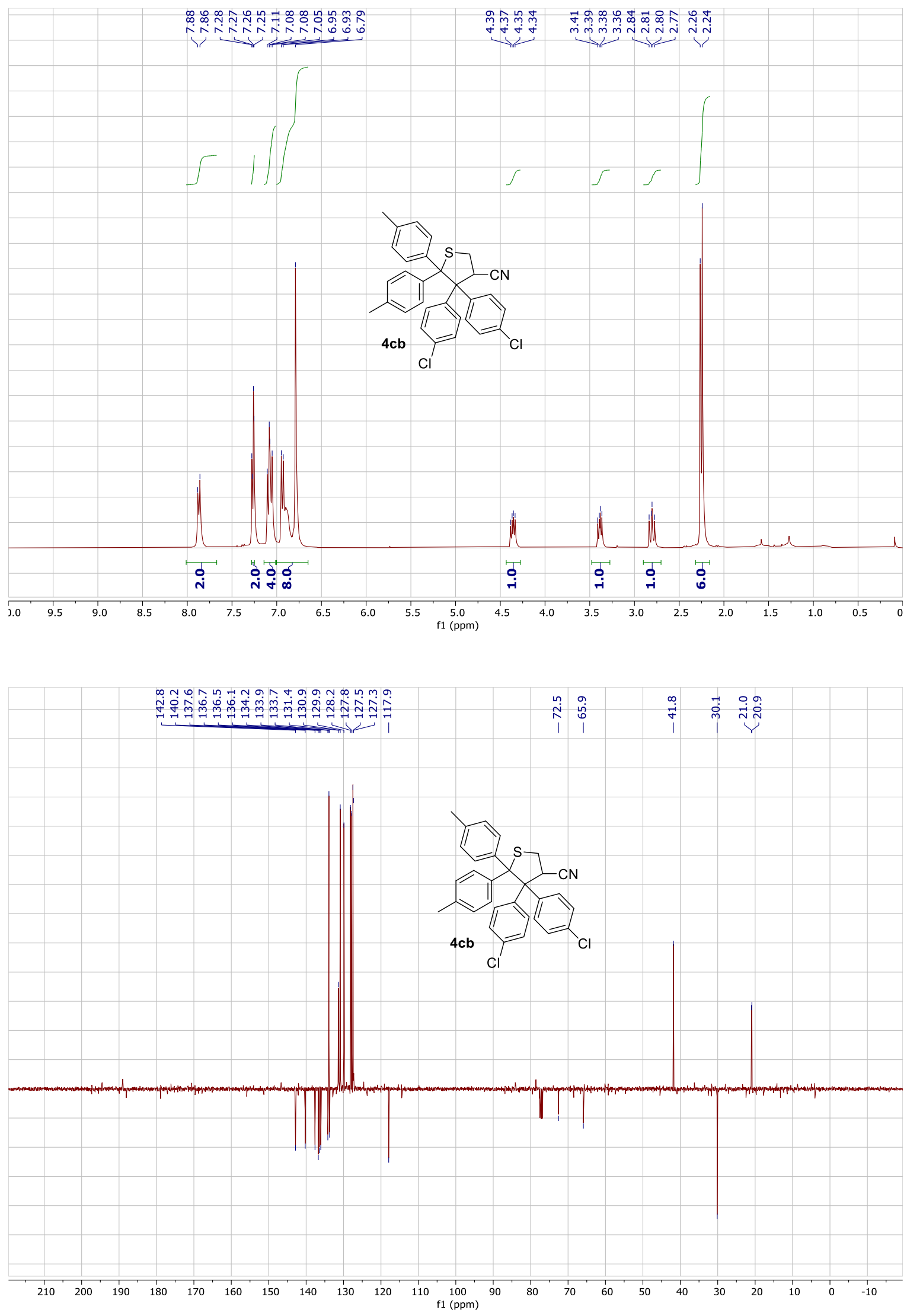
4,4-Diphenyl-5,5-bis(3-(trifluoromethyl)phenyl)tetrahydrothiophene-3-carbonitrile 4af

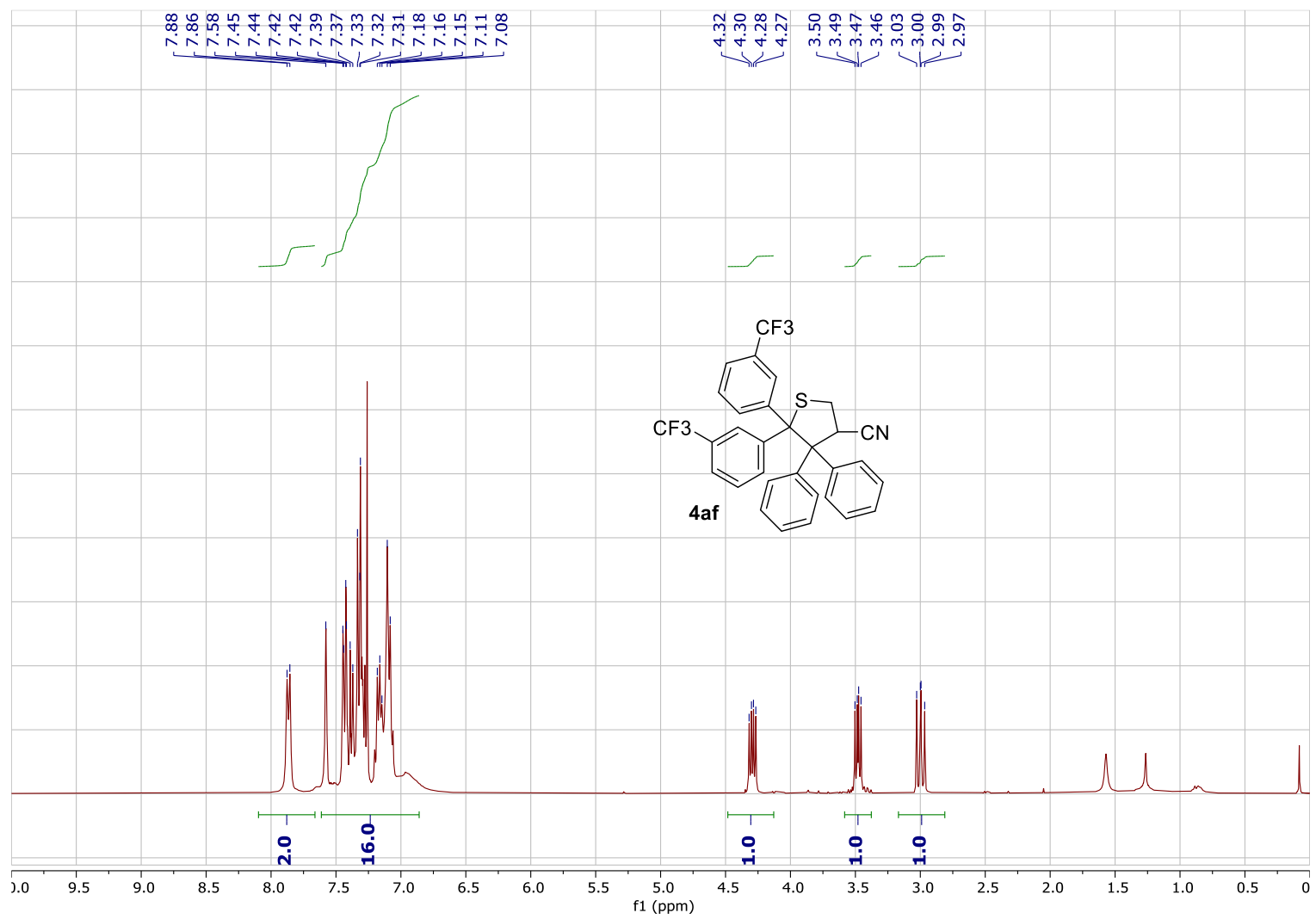

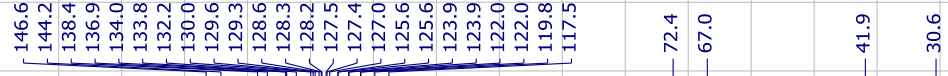

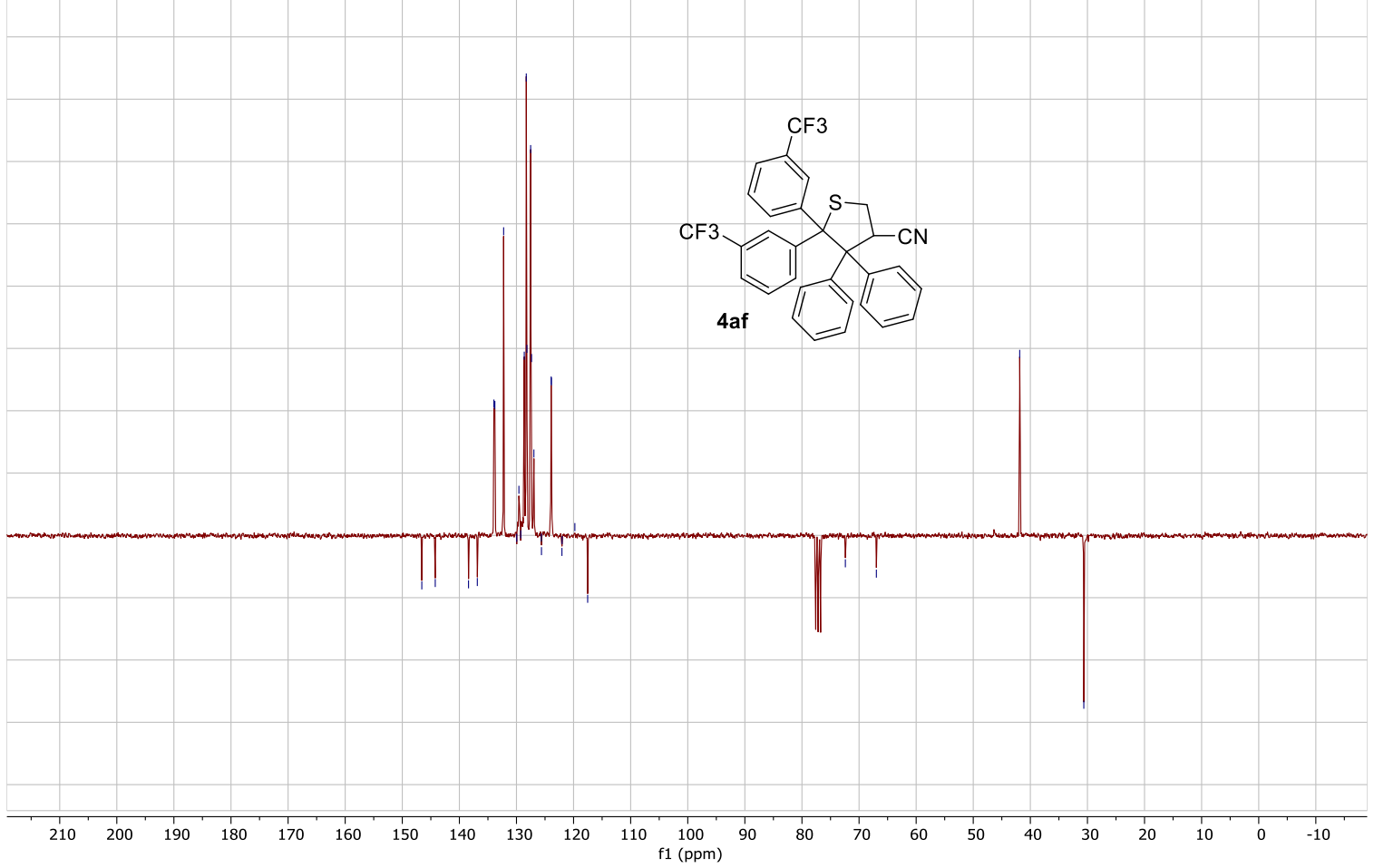


4,4,5-triphenyl-5-(p-tolyl)tetrahydrothiophene-3-carbonitrile 4ag1

(the sample contains the two diastereoisomers 4 ag 1 and 4 ag2 in a 0.5/1 ratio)

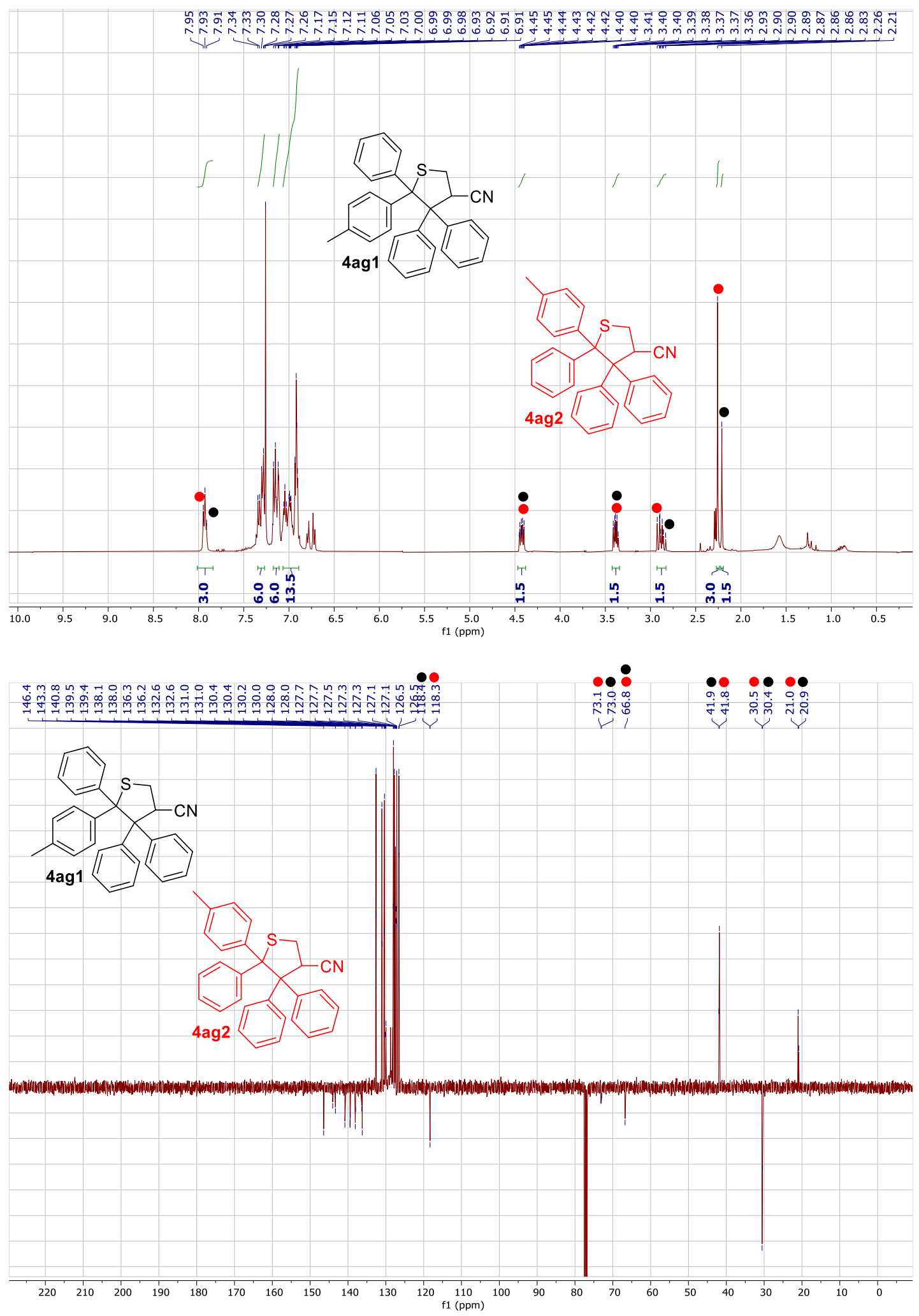


4,4,5-triphenyl-5-(p-tolyl)tetrahydrothiophene-3-carbonitrile 4ag2
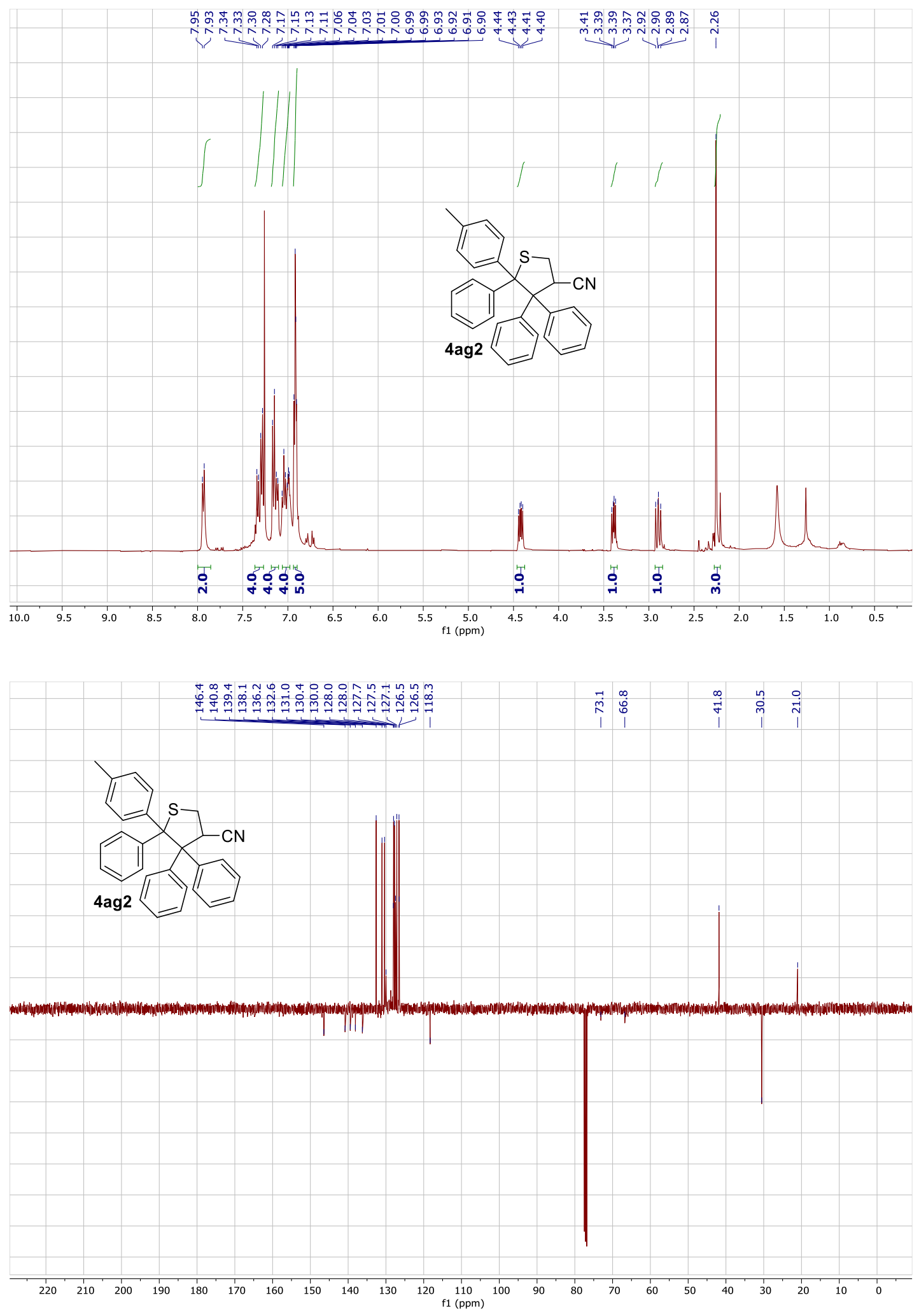


\section{X-ray structure analysis of compounds $3 a, 4 a, 4 e$ and 4ac:}

3a: Compound 3a was dissolved in methanol and left to evaporate slowly to yield colorless needle. A suitable crystal $(0.120 \mathrm{~mm} \times 0.080 \mathrm{~mm} \times 0.025 \mathrm{~mm})$ was selected and mounted on the VENTURE PHOTON100 CMOS Bruker diffractometer.

4a: Compound 4a was dissolved in chloroform and left to evaporate slowly to yield colorless plate. A suitable crystal $(0.310 \mathrm{~mm} \times 0.290 \mathrm{~mm} \times 0.090 \mathrm{~mm})$ was selected and mounted on the Kappa X8 APPEX II Bruker diffractometer.

4ac: Compound 4ac was dissolved in diethyl ether and petroleum ether was diffused slowly to the solution to yield colorless block. A suitable crystal $(0.130 \mathrm{~mm} \times 0.080 \mathrm{~mm} \times 0.030 \mathrm{~mm})$ was selected and mounted on the VENTURE PHOTON100 CMOS Bruker diffractometer.

4e: Compound 4e was dissolved in ethyl acetate and hexane was diffused slowly to the solution to yield colorless block. A suitable crystal $(0.110 \mathrm{~mm} \times 0.090 \mathrm{~mm} \times 0.070 \mathrm{~mm})$ was selected and mounted on the VENTURE PHOTON100 CMOS Bruker diffractometer.

X-ray diffraction data for compounds 3a, 4ac \& 4e were collected by using a VENTURE PHOTON100 CMOS Bruker diffractometer with Micro-focus luS source Mo Ka radiation. X-ray diffraction data for compounds $\mathbf{4 a}$ were collected by using a Kappa X8 APPEX II Bruker diffractometer with graphite-monochromated Moка radiation ( $\lambda=$ $0.71073 \AA$ A). Crystals were mounted on a CryoLoop (Hampton Research) with Paratone-N (Hampton Research) as cryoprotectant and then flashfrozen in a nitrogen-gas stream at $100 \mathrm{~K}$. The temperature of the crystal was maintained at the selected value by means of a 700 series Cryostream cooling device to within an accuracy of $\pm 1 \mathrm{~K}$. The data were corrected for Lorentz polarization, and absorption effects. The structures were solved by direct methods using SHELXS-97 ${ }^{\mathrm{S4}}$ and refined against $F^{2}$ by full-matrix least-squares techniques using SHELXL$2018^{\mathrm{S5}}$ with anisotropic displacement parameters for all non-hydrogen atoms. Hydrogen atoms were located on a difference Fourier map and introduced into the calculations as a riding model with isotropic thermal parameters. All calculations were performed by using the Crystal Structure crystallographic software package WINGX. ${ }^{\mathrm{s} 6}$

\footnotetext{
${ }^{54}$ Sheldrick, G. M. SHELXS-97, Program for Crystal Structure Solution, University of Göttingen, Göttingen, Germany (1997).

${ }^{55}$ Sheldrick, G. M. A short history of SHELX. Acta Cryst. A 2008, 64, 112-122.

${ }^{56}$ Farrugia, L. J. WinGX suite for smallmolecule single-crystal crystallography. J. Appl. Cryst. 1999, 32, 837-838.
} 
For compound 4ac, the presence of solvent molecules (petroleum ether) was evidenced by the residual peaks located in the open channels.

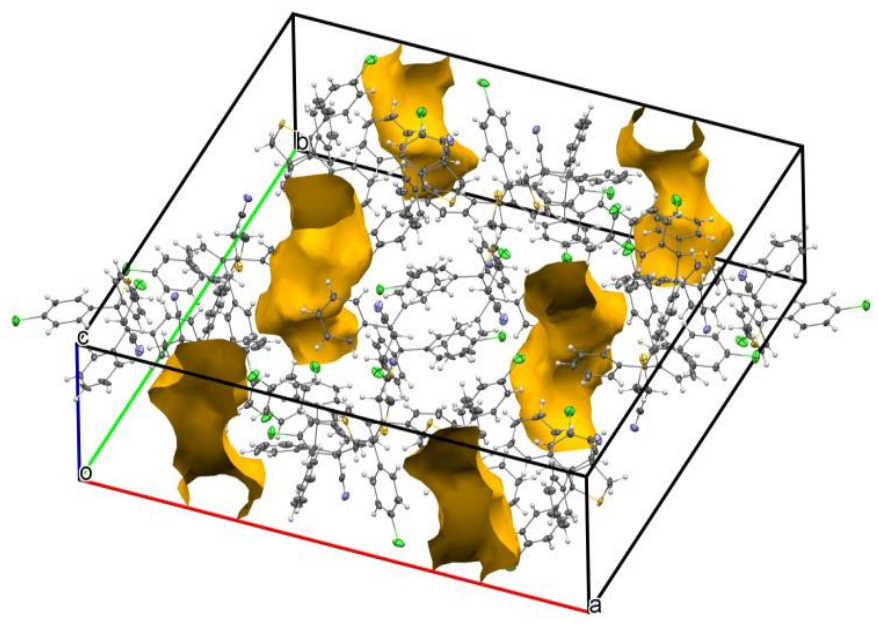

Figure S1: Calculated solvent space for compound 4ac show channel structure of the void space along a axis.

Disorder was such that the system could not be modelled even with restraints. Consequently, SQUEEZE ${ }^{\mathrm{S7}}$ (from PLATON) was used to calculate the void space, the electron count and to obtain a new HKL file. A large number of solvent molecules from petroleum ether (222 electrons) were considered per unit of cell.

Without solvent molecules: $R_{1}=0.0893$ for 8172 reflections of $I>2(I)$ and $w R_{2}=0.2859$ for all data. With solvent molecules (SQUEEZE): R1= 0.0716 for 8172 reflections of $\mathrm{I}>2(\mathrm{I})$ and $\mathrm{WR} 2=0.2448$ for all data, the volume fraction was calculated to $1780 \AA^{3}$ which corresponds to $17 \%$ of the unit cell volume, and to 222 electrons per unit cell allocated to solvent molecules.

The crystal data collection and refinement parameters are given in Table S1.

CCDC 2008236-2008239 contains the supplementary crystallographic data for this paper. These data can be obtained free of charge from the Cambridge Crystallographic Data Centre via http://www.ccdc.cam.ac.uk/Community/.

\footnotetext{
S7 V.d. Sluis P.; Spek, A. L. BYPASS: an effective method for the refinement of crystal structures containing disordered solvent regions. Acta Crystallogr., Sect. A: Found. Crystallogr. 1990, 46, 194-201.
} 
Table S1: Crystallographic data and structure refinement details.

\begin{tabular}{|c|c|c|c|c|}
\hline Compound & $3 a$ & $4 a$ & $4 a c$ & $4 e$ \\
\hline CCDC & 2008236 & 2008237 & 2008239 & 2008238 \\
\hline Empirical Formula & $\mathrm{C}_{16} \mathrm{H}_{13} \mathrm{~N} \mathrm{~S}$ & $\mathrm{C}_{29} \mathrm{H}_{23} \mathrm{~N} \mathrm{~S}$ & $\mathrm{C}_{29} \mathrm{H}_{21} \mathrm{Cl}_{2} \mathrm{~N} \mathrm{~S}$ [+ solvent] & $\mathrm{C}_{29} \mathrm{H}_{19} \mathrm{~F}_{4} \mathrm{~N} \mathrm{~S}$ \\
\hline$M_{r}$ & 251.33 & 417.54 & $486.43[+$ solvent $]$ & 489.51 \\
\hline Crystal size, $\mathrm{mm}^{3}$ & $0.120 \times 0.080 \times 0.025$ & $0.310 \times 0.290 \times 0.090$ & $0.130 \times 0.080 \times 0.030$ & $0.110 \times 0.090 \times 0.070$ \\
\hline Crystal system & orthorhombic & monoclinic & tetragonal & monoclinic \\
\hline Space group & $P \cap a 2_{1}$ & $P 2_{1} / c$ & $/ 4 \sqrt{1} / a$ & $P 2_{1} / c$ \\
\hline a, $\AA$ & $15.7928(9)$ & $9.7499(2)$ & $32.704(3)$ & $10.0687(7)$ \\
\hline$b, \AA$ & $12.1864(7)$ & $9.7270(2)$ & $32.704(3)$ & $10.2539(7)$ \\
\hline C, $\AA$ & $6.6114(3)$ & $22.6969(5)$ & 9.9559(9) & $22.3193(12)$ \\
\hline$\alpha$, & 90 & 90 & 90 & 90 \\
\hline$\beta,{ }^{\circ}$ & 90 & $91.4320(10)$ & 90 & $95.316(2)$ \\
\hline$\gamma_{1}^{\circ}$ & 90 & 90 & 90 & 90 \\
\hline Cell volume, $\AA^{3}$ & $1272.41(12)$ & $2151.84(8)$ & $10648(2)$ & $2294.4(3)$ \\
\hline$Z ; Z^{\prime}$ & $4 ; 1$ & $4 ; 1$ & $16 ; 1$ & $4 ; 1$ \\
\hline $\mathrm{T}, \mathrm{K}$ & $100(1)$ & $100(1)$ & $100(1)$ & $100(1)$ \\
\hline $\begin{array}{c}\text { Radiation type ; } \\
\text { wavelength } \AA\end{array}$ & MoK $\alpha ; 0.71073$ & MoK $\alpha ; 0.71073$ & MoK $\alpha ; 0.71073$ & MoK $\alpha ; 0.71073$ \\
\hline $\mathrm{F}_{000}$ & 528 & 880 & $4032[+$ solvent] & 1008 \\
\hline$\mu, \mathrm{mm}^{-1}$ & 0.234 & 0.167 & 0.339 & 0.192 \\
\hline range $^{\circ}$ & $2.111-32.621$ & $1.795-30.612$ & $2.138-30.585$ & $2.607-32.670$ \\
\hline Reflection collected & 38541 & 45129 & 308735 & 149051 \\
\hline Reflections unique & 4550 & 6611 & 8172 & 8417 \\
\hline $\mathrm{R}_{\text {int }}$ & 0.0835 & 0.0438 & 0.1447 & 0.0846 \\
\hline GOF & 1.024 & 1.030 & 1.002 & 1.037 \\
\hline Refl. obs. $(I>2(I))$ & 3827 & 5326 & 3570 & 6749 \\
\hline Parameters & 163 & 280 & 298 & 316 \\
\hline$w_{2}$ (all data) & 0.0913 & 0.1042 & 0.2448 & 0.1206 \\
\hline $\mathrm{R}$ value $(I>2(I))$ & 0.0390 & 0.0398 & 0.0716 & 0.0392 \\
\hline $\begin{array}{c}\text { Largest diff. peak and } \\
\text { hole }\left(e-. \AA^{-3}\right)\end{array}$ & $0.727 ;-0.259$ & $0.392 ;-0.275$ & $0.469 ;-1.051$ & $0.454 ;-0.383$ \\
\hline
\end{tabular}




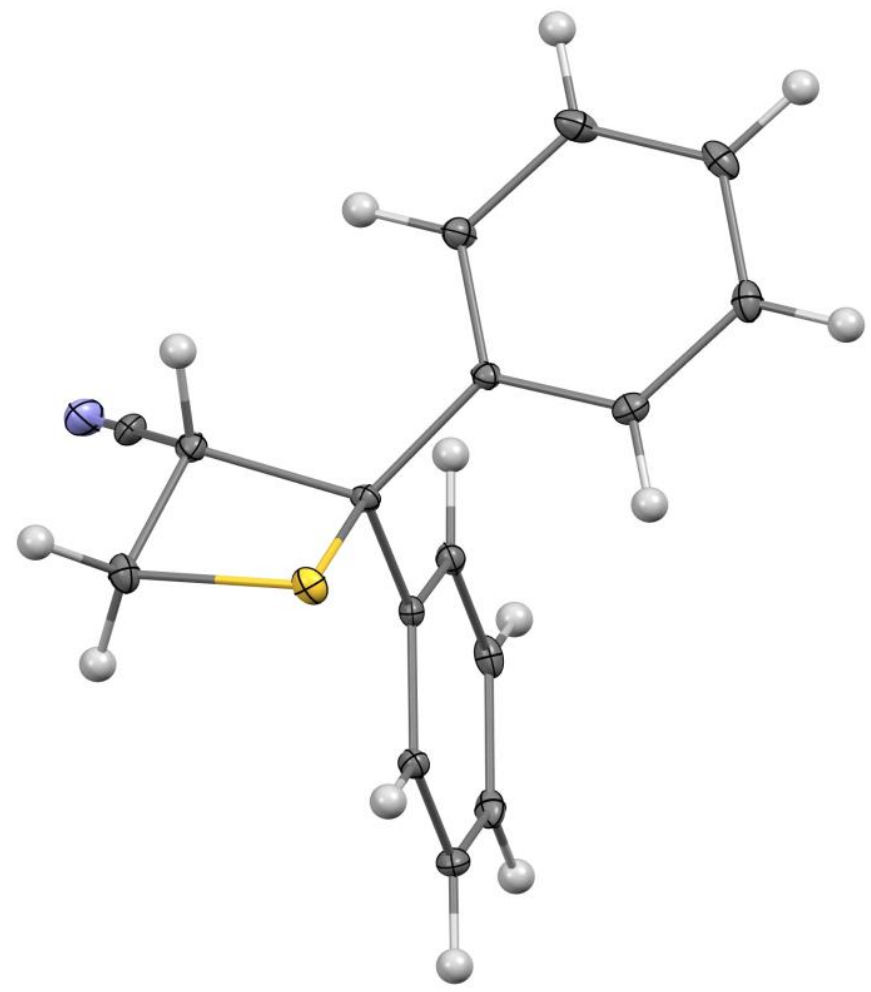

Figure S2: An ORTEP drawing of compound 3a. Thermal ellipsoids are shown at the $30 \%$ level.

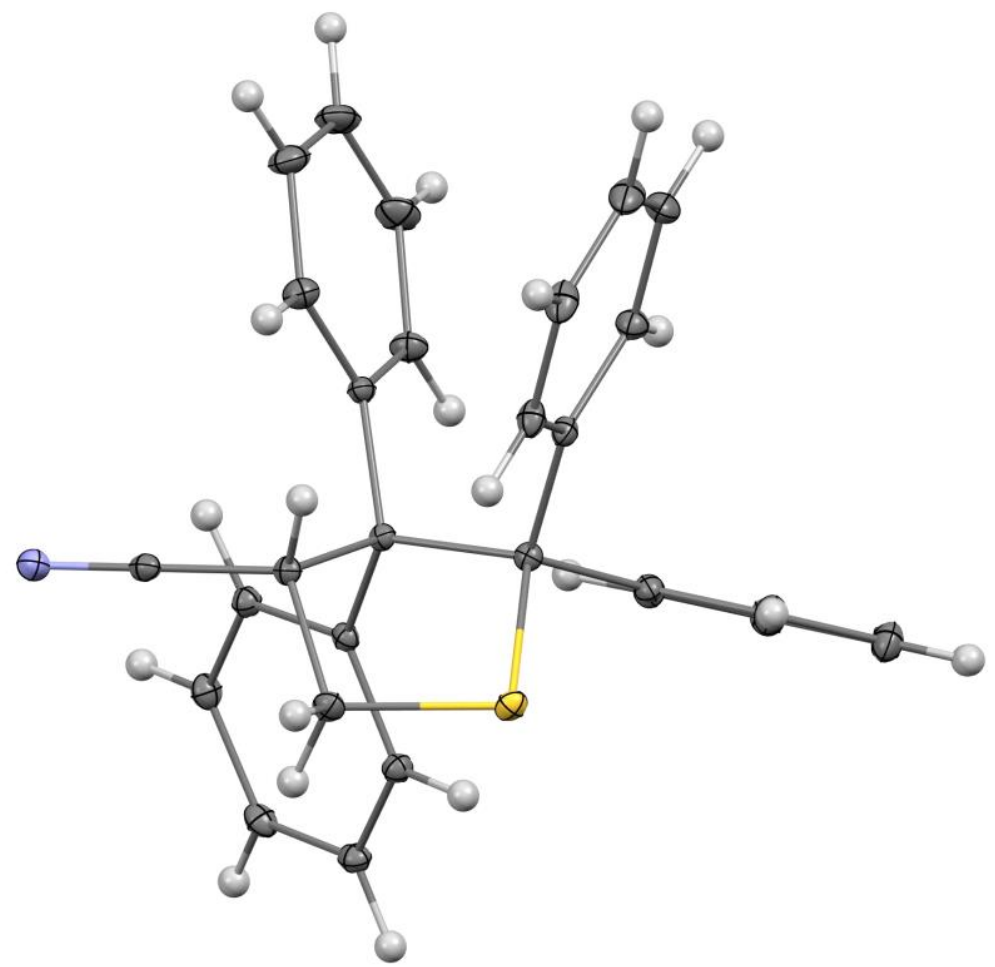

Figure S3: An ORTEP drawing of compound 4a. Thermal ellipsoids are shown at the $30 \%$ level. 


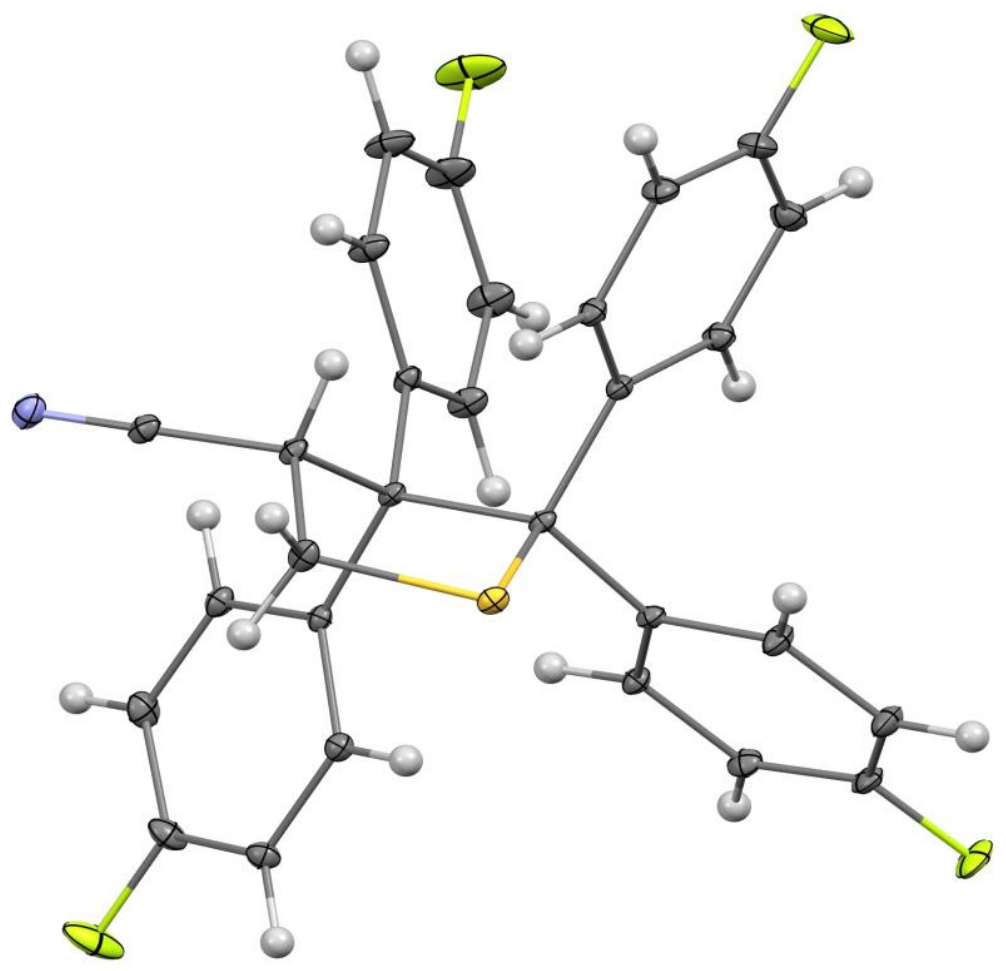

Figure S4: An ORTEP drawing of compound 4e. Thermal ellipsoids are shown at the $30 \%$ level.

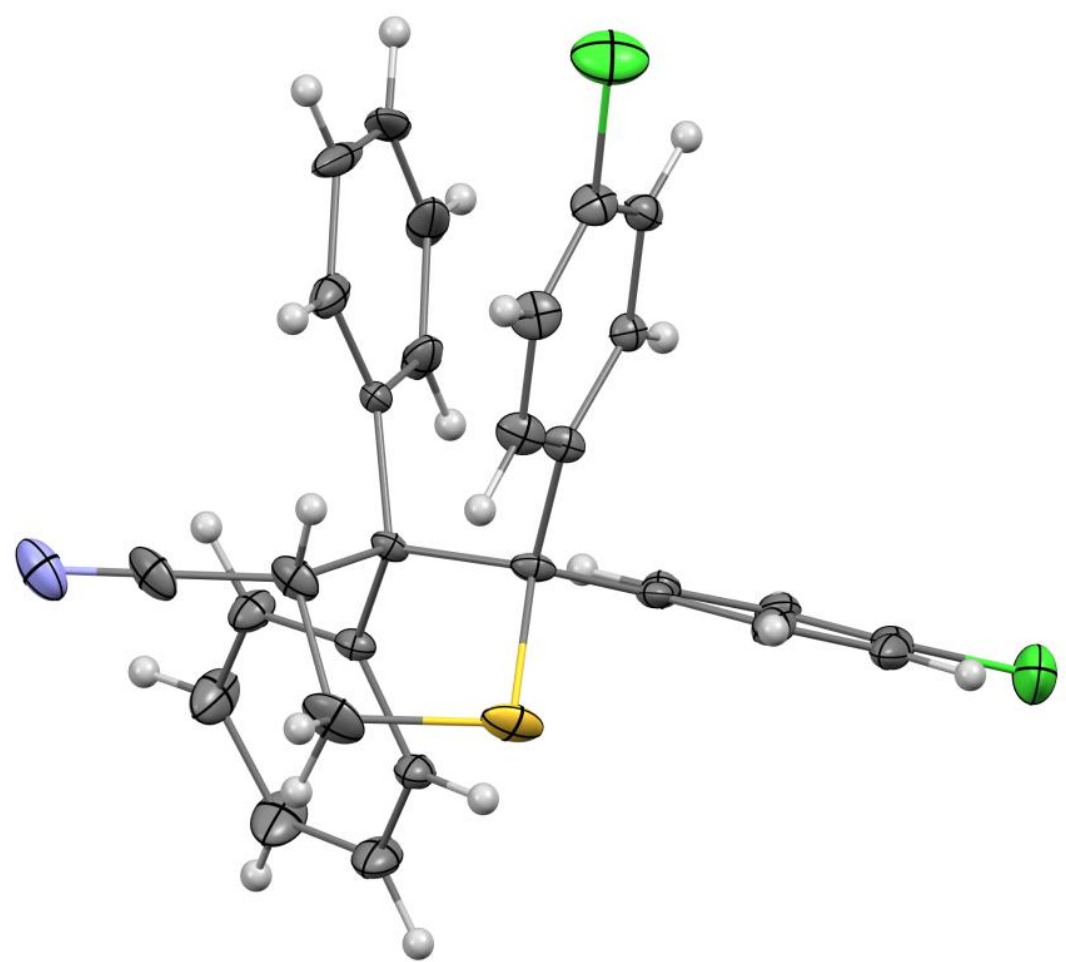

Figure S5: An ORTEP drawing of compound 4ac. Thermal ellipsoids are shown at the $30 \%$ level. 